\title{
REVIEWS
}

\section{Lipid nanoparticles for mRNA delivery}

\author{
Xucheng Hou' ${ }^{1}$, Tal Zaks ${ }^{2 凶}$, Robert Langer $\mathbb{B}^{3,4 凶}$ and Yizhou Dong $\mathbb{1}^{1 凶}$
}

Abstract | Messenger RNA (mRNA) has emerged as a new category of therapeutic agent to prevent and treat various diseases. To function in vivo, mRNA requires safe, effective and stable delivery systems that protect the nucleic acid from degradation and that allow cellular uptake and $m R N A$ release. Lipid nanoparticles have successfully entered the clinic for the delivery of mRNA; in particular, lipid nanoparticle-mRNA vaccines are now in clinical use against coronavirus disease 2019 (COVID-19), which marks a milestone for mRNA therapeutics. In this Review, we discuss the design of lipid nanoparticles for mRNA delivery and examine physiological barriers and possible administration routes for lipid nanoparticle-mRNA systems. We then consider key points for the clinical translation of lipid nanoparticle-mRNA formulations, including good manufacturing practice, stability, storage and safety, and highlight preclinical and clinical studies of lipid nanoparticle-mRNA therapeutics for infectious diseases, cancer and genetic disorders. Finally, we give an outlook to future possibilities and remaining challenges for this promising technology.

'Division of Pharmaceutics \& Pharmacology, College of Pharmacy, The Ohio State University, Columbus, $\mathrm{OH}$, USA.

${ }^{2}$ Moderna, Inc., Cambridge, MA, USA.

${ }^{3}$ David $H$. Koch Institute for Integrative Cancer Research, Massachusetts Institute of Technology, Cambridge, MA, USA.

${ }^{4}$ Department of Chemical Engineering, Massachusetts Institute of Technology, Cambridge, MA, USA.

凶e-mail:tal.zaks@ modernatx.com; rlanger@ mit.edu;dong.525@osu.edu https://doi.org/10.1038/ s41578-021-00358-0
Messenger RNA (mRNA), which was discovered by pioneering studies in 1947-1961 (REF. ${ }^{1}$ ), is a transient intermediator between genes and proteins. By the late 1980s, investigations of mRNA structure and function resulted in the development of in vitro-transcribed (IVT) mRNA $^{2}$. Since the first proof-of-concept animal study in $1990\left(\mathrm{REF}^{3}{ }^{3}\right)$, numerous strategies have been explored to ameliorate the instability and immunogenicity of IVT mRNA ${ }^{2,4}$. Additionally, advances in drug delivery systems have expedited the preclinical development of mRNA therapeutics ${ }^{5-17}$, providing the basis for mRNA as a new class of drug (FIG. 1).

mRNA has shown therapeutic potential in a range of applications, including viral vaccines, protein replacement therapies, cancer immunotherapies, cellular reprogramming and genome editing ${ }^{2,4,7-17}$. To achieve therapeutic effects, mRNA molecules have to reach specific target cells and produce sufficient proteins of interest. However, targeted delivery and endosomal escape remain challenging for mRNA delivery systems, highlighting the need for safe and effective mRNA delivery materials.

A variety of materials have been developed for mRNA delivery, including lipids, lipid-like materials, polymers and protein derivatives ${ }^{7-17}$. In particular, lipid nanoparticles have been thoroughly investigated and successfully entered the clinic for the delivery of small molecules ${ }^{18}$, siRNA drugs ${ }^{18}$ and mRNA $^{19-21}$. Notably, two authorized coronavirus disease 2019 (COVID-19) vaccines, mRNA$1273\left(\right.$ REFS $\left.^{19,20}\right)$ and BNT162 ${ }^{21}$, use lipid nanoparticles to deliver antigen mRNA. Many other lipid nanoparticlemRNA formulations have been developed and are under clinical evaluation for the prevention and treatment of virus infections, cancer and genetic diseases ${ }^{7-17}$ (TABLES 1,2).

In this Review, we briefly overview representative lipid nanoparticles used for mRNA delivery and describe key steps in the preclinical development of lipid nanoparticle-mRNA formulations, including the overcoming of physiological barriers, different administration routes, manufacturing and safety profiles. Finally, we highlight important examples of lipid nanoparticle-mRNA formulations in clinical studies and provide future perspectives for lipid nanoparticles and mRNA therapeutics.

\section{Development of lipids for mRNA delivery}

In 1976, nucleic acids were encapsulated and delivered in polymeric particles ${ }^{5}$. Later, exogenous mRNA delivery into host cells was demonstrated with liposomes ${ }^{22,23}$ (FIG. 1). Lipids are amphiphilic molecules that contain three domains: a polar head group, a hydrophobic tail region and a linker between the two domains. Cationic lipids, ionizable lipids and other types of lipid have been explored for mRNA delivery (FIG. 2).

Cationic lipids. Cationic lipids have a head group with permanent positive charges ${ }^{11,14}$. For example, 1,2di-O-octadecenyl-3-trimethylammonium-propane (DOTMA), a quaternary ammonium lipid, has been applied for mRNA delivery in multiple cell types ${ }^{24}$, and was commercialized as Lipofectin in combination with 1,2-dioleoyl-sn-glycero-3-phosphoethanolamine $(\mathrm{DOPE})^{24}$. 1,2-dioleoyl-3-trimethylammonium-propane (DOTAP), a biodegradable analogue of DOTMA, was 


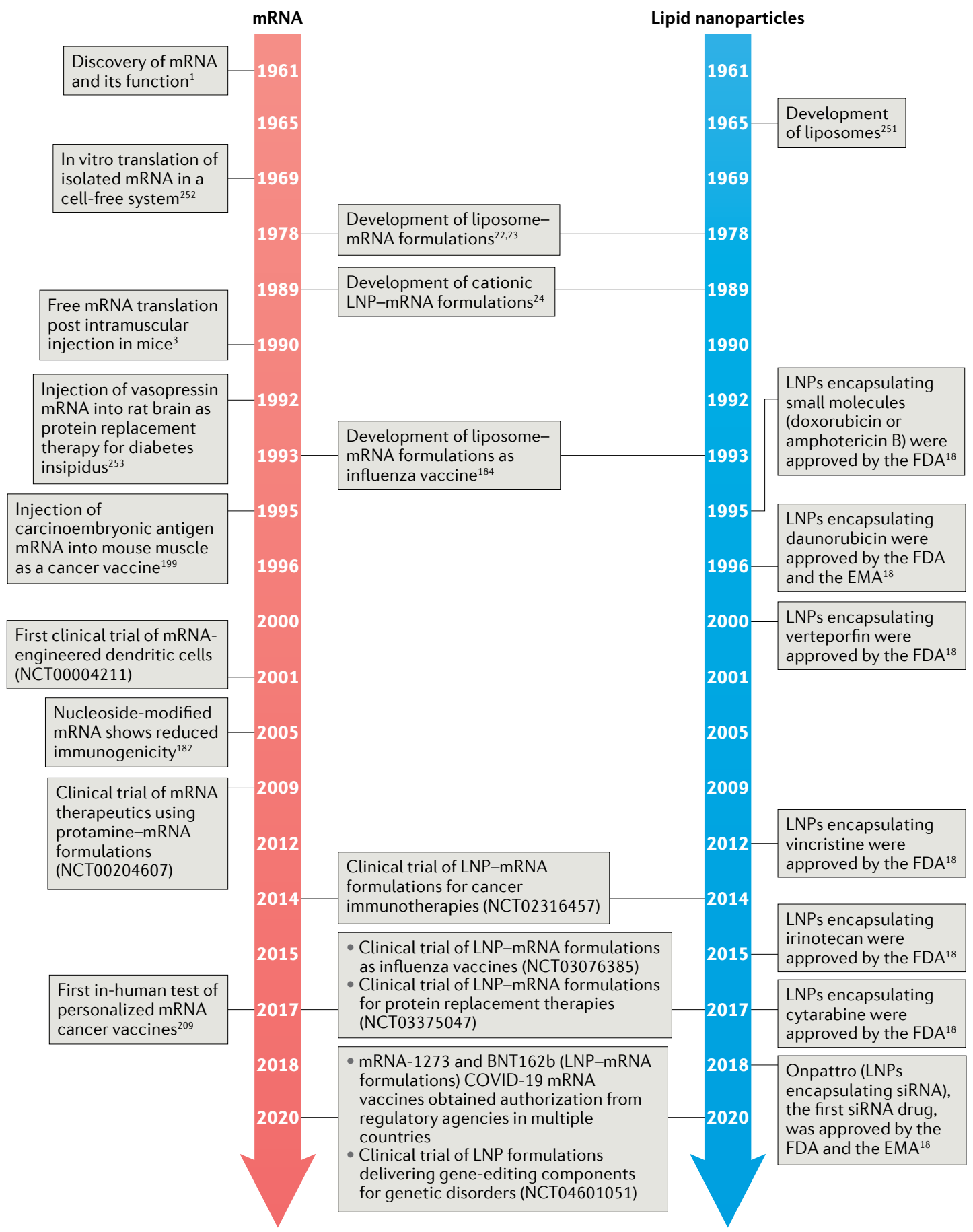

Fig. 1 | Timeline of some key milestones for mRNA and lipid nanoparticle development. COVID-19, coronavirus disease 2019; EMA, European Medicines Agency; FDA, United States Food and Drug Administration; LNP, lipid nanoparticle ${ }^{251-253}$.

also studied for mRNA delivery ${ }^{25}$, and is part of the commercial agent MegaFectin, together with DOPE or cholesterol. DOTMA and DOTAP have both been applied either alone or combined with other materials for mRNA delivery ${ }^{7-17}$; for example, spleen-targeted DOTMA-mRNA lipoplexes (RNA-LPX) have been developed as systemic cancer vaccine ${ }^{26}$. The same formulation has also been designed as mRNA vaccine for the treatment of autoimmune encephalomyelitis ${ }^{27}$. This vaccine induces the proliferation of antigen-specific
$\mathrm{CD}^{+}$regulatory $\mathrm{T}$ cells, leading to enhanced immunosuppression and a reduction of clinical symptoms in mouse models ${ }^{27}$. DOTAP-based cationic nanoemulsions can deliver antigen mRNA against viral, bacterial and parasitic infections ${ }^{28-31}$. Moreover, DOTAP-polymer hybrid nanoparticles can deliver mRNA molecules for the treatment of cancer ${ }^{32-37}$, infections ${ }^{38-41}$ and genetic disorders ${ }^{42}$. Incorporating carbonate apatite in DOTAP-based lipid nanoparticles increases the interaction between the particles and cellular membranes ${ }^{43}$. 
The delivery efficiency can further be improved by conjugating fibronectin to the lipid nanoparticles, which is a cellular adhesion protein accelerating the endocytic rate $^{44}$.

Dimethyldioctadecylammonium bromide (DDAB), a quaternary ammonium lipid, can not only from complexes with mRNA but also stimulate innate immune responses ${ }^{45}$, thereby serving as immune adjuvant for mRNA vaccines ${ }^{46,47}$. DDAB and DOPE constitute the commercial product TransfectAce. The commercialized agent Lipofectamine is composed of DOPE and 2,3-dioleyloxy- $N$-[2-(sperminecarboxamido) ethyl]-N,N-dimethyl-1-propanaminium trifluoroacetate (DOSPA), a cationic lipid containing quaternary

Table 1 | Representative clinical trials of lipid nanoparticle-mRNA vaccines against infections and cancer

\begin{tabular}{|c|c|c|c|c|c|}
\hline Name & Disease & Encoded antigen & $\begin{array}{l}\text { Administration } \\
\text { route }\end{array}$ & $\begin{array}{l}\text { ClinicalTrials.gov } \\
\text { identifier }\end{array}$ & Phase \\
\hline \multicolumn{6}{|l|}{ Infections } \\
\hline mRNA-1273 & SARS-CoV-2 & Spike & i.m. & NCT04470427 & $\begin{array}{l}\text { III (EUA } \\
\text { and CMA) }\end{array}$ \\
\hline BNT162b2 & SARS-CoV-2 & Spike & i.m. & NCT04368728 & $\begin{array}{l}\text { III (EUA } \\
\text { and CMA) }\end{array}$ \\
\hline $\mathrm{CVnCoV}$ & SARS-CoV-2 & Spike & i.m. & NCT04652102 & III \\
\hline LNP-nCoVsaRNA & SARS-CoV-2 & Spike & i.m. & ISRCTN17072692 & I \\
\hline ARCT-021 & SARS-CoV-2 & Spike & i.m. & NCT04728347 & II \\
\hline ARCoV & SARS-CoV-2 & $\begin{array}{l}\text { Receptor-binding } \\
\text { domain }\end{array}$ & i.m. & ChiCTR2000034112 & I \\
\hline mRNA-1440 & Influenza H10N8 & Haemagglutinin & i.m. & NCT03076385 & I \\
\hline mRNA-1851 & Influenza H7N9 & Haemagglutinin & i.m. & NCT03345043 & I \\
\hline mRNA-1893 & Zika virus & $\begin{array}{l}\text { Pre-membrane } \\
\text { and envelope } \\
\text { glycoproteins }\end{array}$ & i.m. & NCT04064905 & I \\
\hline mRNA-1345 & $\begin{array}{l}\text { Respiratory syncytial } \\
\text { virus }\end{array}$ & F glycoprotein & i.m. & NCT04528719 & I \\
\hline mRNA-1653 & $\begin{array}{l}\text { Metapneumovirus } \\
\text { and parainfluenza } \\
\text { virus type } 3 \text { (MPV/ } \\
\text { PIV3) }\end{array}$ & $\begin{array}{l}\text { MPV and PIV3 F } \\
\text { glycoproteins }\end{array}$ & i.m. & NCT03392389 & I \\
\hline mRNA-1647 & Cytomegalovirus & $\begin{array}{l}\text { Pentameric complex } \\
\text { and B glycoprotein }\end{array}$ & i.m. & NCT04232280 & II \\
\hline mRNA-1388 & Chikungunya virus & $\begin{array}{l}\text { Chikungunya virus } \\
\text { antigens }\end{array}$ & i.m. & NCT03325075 & I \\
\hline CV7202 & Rabies virus & Gglycoprotein & i.m. & NCT03713086 & I \\
\hline \multicolumn{6}{|l|}{ Cancer } \\
\hline $\begin{array}{l}\text { mRNA-5671/ } \\
\text { V941 }\end{array}$ & $\begin{array}{l}\text { Non-small-cell lung } \\
\text { cancer, colorectal } \\
\text { cancer, pancreatic } \\
\text { adenocarcinoma }\end{array}$ & KRAS antigens & i.m. & NCT03948763 & I \\
\hline mRNA-4157 & Melanoma & $\begin{array}{l}\text { Personalized } \\
\text { neoantigens }\end{array}$ & i.m. & NCT03897881 & ॥ \\
\hline mRNA-4650 & $\begin{array}{l}\text { Gastrointestinal } \\
\text { cancer }\end{array}$ & $\begin{array}{l}\text { Personalized } \\
\text { neoantigens }\end{array}$ & i.m. & NCT03480152 & $I / I I$ \\
\hline FixVac & Melanoma & $\begin{array}{l}\text { NY-ESO-1, tyrosinase, } \\
\text { MAGE-A3, TPTE }\end{array}$ & i.v. & NCT02410733 & I \\
\hline TNBC-MERIT & $\begin{array}{l}\text { Triple-negative } \\
\text { breast cancer }\end{array}$ & $\begin{array}{l}\text { Personalized } \\
\text { neoantigens }\end{array}$ & i.v. & NCT02316457 & I \\
\hline HARE-40 & HPV-positive cancers & $\begin{array}{l}\text { HPV oncoproteins E6 } \\
\text { and E7 }\end{array}$ & i.d. & NCT03418480 & I/II \\
\hline RO7198457 & Melanoma & $\begin{array}{l}\text { Personalized } \\
\text { neoantigens }\end{array}$ & i.v. & NCT03815058 & II \\
\hline W_ova1 & Ovarian cancer & $\begin{array}{l}\text { Ovarian cancer } \\
\text { antigens }\end{array}$ & i.v. & NCT04163094 & I \\
\hline
\end{tabular}

CMA, conditional marketing authorization; EUA, Emergency Use Authorization; HPV, human papillomavirus; i.d., intradermal; i.m., intramuscular; i.v., intravenous; KRAS, Kirsten rat sarcoma 2 viral oncogene homologue; MAGE-A3, melanoma antigen family A; NY-ESO-1, New York esophageal squamous cell carcinoma 1; SARS-CoV-2, severe acute respiratory syndrome coronavirus 2; TPTE, putative tyrosine-protein phosphatase. 
Table 2 | Representative clinical trials of lipid nanoparticle-mRNA therapeutics against infections, cancer and genetic disorders

\begin{tabular}{|c|c|c|c|c|c|}
\hline Name & Disease & Encoded protein & $\begin{array}{l}\text { Administration } \\
\text { route }\end{array}$ & $\begin{array}{l}\text { ClinicalTrials. } \\
\text { gov identifier }\end{array}$ & Phase \\
\hline \multicolumn{6}{|l|}{ Infections } \\
\hline mRNA-1944 & Chikungunya virus & $\begin{array}{l}\text { Antibody against } \\
\text { chikungunya virus }\end{array}$ & i.v. & NCT03829384 & I \\
\hline \multicolumn{6}{|l|}{ Cancer } \\
\hline mRNA 2416 & Solid tumours & OX40L & Intratumour & NCT03323398 & ॥ \\
\hline mRNA-2752 & Solid tumours & 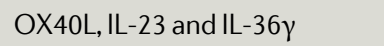 & Intratumour & NCT03739931 & I \\
\hline MEDI1191 & Solid tumours & $\mathrm{IL}-12$ & Intratumour & NCT03946800 & I \\
\hline SAR441000 & Solid tumours & $\begin{array}{l}\text { IL-12sc, IL-15sushi, IFNa and } \\
\text { GM-CSF }\end{array}$ & Intratumour & NCT03871348 & I \\
\hline \multicolumn{6}{|c|}{ Genetic disorders } \\
\hline mRNA-3704 & $\begin{array}{l}\text { Methylmalonic } \\
\text { acidaemia }\end{array}$ & Methylmalonyl-CoA mutase & i.v. & NCT03810690 & $\mathrm{I} / \mathrm{II}$ \\
\hline mRNA-3927 & Propionic acidaemia & Propionyl-CoA carboxylase & i.v. & NCT04159103 & $\mathrm{I} / \mathrm{II}$ \\
\hline MRT5201 & $\begin{array}{l}\text { Ornithine } \\
\text { transcarbamylase } \\
\text { deficiency }\end{array}$ & Ornithine transcarbamylase & i.v. & NCT03767270 & $\mathrm{I} / \mathrm{II}$ \\
\hline MRT5005 & Cystic fibrosis & $\begin{array}{l}\text { Cystic fibrosis transmembrane } \\
\text { conductance regulator }\end{array}$ & Inhalation & NCT03375047 & $\mathrm{I} / \mathrm{II}$ \\
\hline NTLA-2001 & $\begin{array}{l}\text { Transthyretin amyloidosis } \\
\text { with polyneuropathy }\end{array}$ & $\begin{array}{l}\text { CRISPR-Cas9 gene editing } \\
\text { system }\end{array}$ & i.v. & NCT04601051 & I \\
\hline
\end{tabular}

ammonium and spermine. Lipofectamine protocols have been optimized to deliver mRNA in diverse cell types, including alveolar cells, cardiac muscle cells and pluripotent stem cells ${ }^{48-50} \cdot 2-((((3 S, 8 S, 9 S, 10 R, 13 R, 14 S$, $17 R)$-10,13-dimethyl-17-((R)-6-methylheptan-2-yl)$2,3,4,7,8,9,10,11,12,13,14,15,16,17$-tetradecahydro-1 $H$-cyclopenta[a]phenanthren-3-yl)oxy)carbonyl)amino)$N, N$-bis(2-hydroxyethyl)- $N$-methylethan-1-aminium bromide (BHEM-Cholesterol) was developed by modifying the head structure of $3 \beta-\left[N-\left(N^{\prime}, N^{\prime}-\right.\right.$ dimethylaminoethane)-carbamoyl]cholesterol (DC-Cholesterol) with hydroxyl groups to improve fusion with cellular membranes ${ }^{51}$. Lipid nanoparticles containing BHEM-Cholesterol have been applied to deliver mRNA encoding clustered regularly interspaced short palindromic repeats (CRISPR)-CRISPR-associated protein 9 (CRISPR-Cas9) and tumour antigens ${ }^{52,53}$. Ethylphosphatidylcholine (ePC) was synthesized by introducing a third alkyloxy group into phosphatidylcholines to eliminate their negative charge. ePC-based lipid nanoparticles have been applied for mRNA-based cancer immunotherapies ${ }^{54,55}$ and protein replacement therapies $^{56}$.

Ionizable lipids. Ionizable lipids are protonated at low $\mathrm{pH}$, which makes them positively charged, but they remain neutral at physiological $\mathrm{pH}\left(\mathrm{REFS}^{7,11,14}\right)$. The $\mathrm{pH}$-sensitivity of ionizable lipids is beneficial for mRNA delivery in vivo, because neutral lipids have less interactions with the anionic membranes of blood cells and, thus, improve the biocompatibility of lipid nanoparticles ${ }^{7,11,14}$. Trapped in endosomes, in which the $\mathrm{pH}$ is lower than in the extracellular environment, ionizable lipids are protonated and, therefore, become positively charged, which may promote membrane destabilization and facilitate endosomal escape of the nanoparticles ${ }^{7,11,14}$ Ionizable lipids originally developed for DNA transfection, such as (2S)-2,5-bis(3aminopropylamino)- $\mathrm{N}$-[2-(dioctadecylamino)acetyl] pentanamide (DOGS; Transfectam $)^{57}, N^{1}-[2-((1 S)-1-[(3-$ aminopropyl)amino]-4-[di(3-aminopropyl)amino] butylcarboxamido)ethyl]-3,4-di[oleyloxy]-benzamide (MVL5) $^{58}$, DC-Cholesterol ${ }^{59}$ and $N^{4}$-cholesterylspermine (GL67) ${ }^{60}$, have also been explored for mRNA delivery ${ }^{25,61-63}$.

The ionizable lipid 1,2-dilinoleyloxy-N, $\mathrm{N}$-dimethyl-3-aminopropane (DLin-DMA) was initially synthesized for siRNA delivery ${ }^{64}$, and delivery efficacy was improved by modification of the linker and hydrophobic regions, resulting in 2,2-dilinoleyl-4-dimethylaminoethyl-[1,3]-dioxolane (DLin-KC2-DMA) ${ }^{65}$. Further optimization of the amine head group of DLin-KC2-DMA led to (6Z,9Z,28Z,31Z)-heptatriaconta-6,9,28,31-tetraen-19-yl 4-(dimethylamino) butanoate (DLin-MC3-DMA; MC3), which is a key delivery component of Onpattro, the first United States Food and Drug Administration (FDA)-approved siRNA drug ${ }^{18,66}$. MC3-based lipid nanoparticles have also been tested for mRNA therapeutics, such as protein replacement therapies ${ }^{56,67-72}$ and antiviral therapies ${ }^{73-75}$. Incorporation of biodegradable lipids improves the tolerability of lipid nanoparticles, by allowing fast metabolism while retaining mRNA delivery efficacy. The biodegradability of lipids 
can be increased by introducing ester motifs; for example, introducing ester bonds in the linker and lipidic tails of MC3 results in the lipid $\operatorname{di}((Z)$-non-2-en-1-yl) 9-((4-(dimethylamino)butanoyl)oxy)heptadecanedioate $(\mathrm{L} 319)^{76}$, which shows better delivery efficacy and faster elimination from the liver and plasma in vivo in comparison with MC3 $\left(\mathrm{REF}^{76}\right)$. Similarly, the biodegradable lipids heptadecan-9-yl 8-((2-hydroxyethyl) (8-(nonyloxy)-8-oxooctyl)amino)octanoate (Lipid 5) ${ }^{77}$, heptadecan-9-yl 8-((2-hydroxyethyl)(6-oxo-6-(undecyloxy)hexyl)amino) octanoate (Lipid H (SM-102)) $)^{78}$ and ((4-hydroxybutyl)azanediyl)bis(hexane-6,1-diyl) bis(2-hexyldecanoate) (ALC-0315 $)^{79}$ have better in vivo delivery efficacy and pharmacokinetics than MC3. Of note, SM-102 and ALC-0315 are the ionizable delivery components in the mRNA-1273 and BNT162b COVID-19 vaccines, respectively ${ }^{17}$. Biodegradable lipids can also be made of both ester and disulfide motifs ${ }^{80-85}$. Cleavage of the disulfide bonds then drives an intraparticle nucleophilic attack on the ester linker, accelerating their degradation ${ }^{80-85}$.

A combinatorial library has been designed that contains lipid-like materials with different hydrophilic groups and multiple lipidic tails, highlighting the chemical diversity of ionizable lipids ${ }^{86}$. Many lipid-like materials, such as $1,1^{\prime}-((2-(4-(2-((2-)$ (bis(2-hydroxydodecyl) amino)ethyl) (2-hydroxydodecyl)amino)ethyl) piperazin-1-yl)ethyl)azanediyl) bis(dodecan-2-ol) $(\mathrm{C} 12-200)^{87}$, tetrakis(8-methylnonyl) 3,3',3", $3^{\prime \prime \prime}$ (((methylazanediyl) bis(propane-3,1 diyl))bis (azanetriyl))tetrapropionate $\left(306 \mathrm{O}_{\mathrm{i10}}\right)^{88}$ and 3,6-bis(4(bis(2-hydroxydodecyl)amino)butyl)piperazine2,5-dione (cKK-E12) ${ }^{89}$, have been developed to deliver mRNA molecules in vivo ${ }^{90-100}$. For example, cKKE12-based lipid nanoparticles are applied in cancer immunotherapies ${ }^{94,95}$ and genome editing ${ }^{96}$. Replacing the lipidic chains of cKK-E12 with alkenyl amino alcohols results in 3,6-bis(4-(bis ( $9 Z, 12 Z)$-2-hydroxyoctadeca9,12-dien-1-yl)amino)butyl)piperazine-2,5-dione(OF-02), which improves mRNA delivery efficacy in vivo, compared with cKK-E12 (REF. $\left.{ }^{101}\right)$. Further altering the linkage of OF-02 leads to (( 3,6 -dioxopiperazine-2,5-diyl)bis (butane-4,1-diyl))bis(azanetriyl))tetrakis(ethane2,1-diyl) (9Z,9' Z,9" $\left.Z, 9^{\prime \prime \prime} Z, 12 Z, 12^{\prime} Z, 12^{\prime \prime} Z, 12^{\prime \prime \prime} Z\right)$ tetrakis (octadeca-9,12-dienoate) (OF-Deg-Lin) and (((3,6-dioxopiperazine-2,5-diyl)bis(butane-4,1-diyl)) bis(azanetriyl))tetrakis (butane-4,1-diyl) $\left(9 Z, 9^{\prime} Z, 9^{\prime \prime} Z, 9^{\prime \prime \prime} Z\right.$, $12 Z, 12^{\prime} Z, 12^{\prime \prime} Z, 12^{\prime \prime \prime} Z$ )-tetrakis (octadeca-9,12-dienoate) (OF-C4-Deg-Lin), which allow selective delivery of mRNA into the spleen ${ }^{102,103}$. The lipid-like material $N^{1}, N^{3}, N^{5}$-tris(3-(didodecylamino)propyl)benzene1,3,5-tricarboxamide (TT3) can deliver mRNA molecules encoding human factor IX ${ }^{104}$, CRISPR-Cas9 (REF. ${ }^{105}$ ), an interleukin-12 (IL-12) replicon ${ }^{106}$ and severe acute respiratory syndrome coronavirus 2 (SARSCoV-2) antigens ${ }^{107}$. Hexa(octan-3-yl) 9,9', $9^{\prime \prime}, 9^{\prime \prime \prime}, 9^{\prime \prime \prime}, 9^{\prime \prime \prime \prime}$ (((benzene-1,3,5-tricarbonyl)ris(azanediyl)) tris (propane-3,1-diyl))tris(azanetriyl))hexanonanoate (FTT5), which is a biodegradable analogue of TT3, further improves the in vivo delivery efficacy of mRNA encoding human factor VIII and base editing components ${ }^{108}$. In addition, a series of aminoglycoside-derived lipids have been synthesized as mRNA delivery materials ${ }^{109-112}$.

Zwitterionic ionizable lipids can also be applied for mRNA delivery ${ }^{56,113-116}$; for example, lipids composed of a pH-switchable zwitterion and three hydrophobic tails assemble into a cone in the endosomal acidic environment, enabling membrane hexagonal transformation and allowing them to leave the endosome. Thus, lipid nanoparticle-mRNA formulations based on zwitterionic ionizable lipids can escape the endosome, leading to efficient protein expression and genome editing in vivo ${ }^{114}$. In addition to functioning as a delivery component, lipids can have therapeutic effects synergistic with mRNA-encoded proteins ${ }^{117-119}$. For example, lipids with a heterocyclic amine as head group can activate the stimulator of interferon genes (STING) signalling pathway in dendritic cells ${ }^{117}$. These lipids, as part of an mRNA vaccine, induce potent cytolytic T lymphocyte responses and inhibit tumour growth in mouse models ${ }^{117}$. Paclitaxel-conjugated lipids encapsulating tumour suppressor mRNA can be applied to integrate chemotherapy and gene therapy for triple-negative breast cancer ${ }^{118}$.

Other types of lipid. In addition to cationic or ionizable lipids, lipid nanoparticle-mRNA formulations typically contain other lipid components, such as phospholipids (for example, phosphatidylcholine and phosphatidylethanolamine), cholesterol or polyethylene glycol (PEG)-functionalized lipids (PEG-lipids) ${ }^{7,14,17}$. These lipids can improve nanoparticle properties, such as particle stability, delivery efficacy, tolerability and biodistribution ${ }^{7,14,17}$. For example, 1,2-distearoyl-snglycero-3-phosphocholine (DSPC), a phosphatidylcholine with saturated tails, has a melting temperature of $\sim 54^{\circ} \mathrm{C}$ and a cylindrical geometry that allows DSPC molecules to form a lamellar phase, which stabilizes the structure of lipid nanoparticles ${ }^{120}$. DSPC has been used in the mRNA-1273 and BNT162b2 COVID-19 vaccines $^{17}$. DOPE is a phosphoethanolamine with two unsaturated tails, which has a melting temperature of $\sim 30^{\circ} \mathrm{C}$ and a conical shape ${ }^{120}$. DOPE tends to adopt an inverted hexagonal $\mathrm{H}$ (II) phase, which destabilizes endosomal membranes and facilitates endosomal escape of lipid nanoparticles ${ }^{90,120}$. Using DNA barcode-labelled oligonucleotides, the distribution of different lipid nanoparticle formulations can be analysed in a highthroughput manner in vivo ${ }^{121}$, for example, to quantify targeted delivery of nucleic acids in multiple tissues ${ }^{121}$. Based on this method, a series of phosphatidylcholines containing constrained adamantyl groups has been explored for mRNA delivery, including analysis of distribution in different cell types ${ }^{122}$.

Cholesterol can enhance particle stability by modulating membrane integrity and rigidity ${ }^{7,14,17}$. The molecular geometry of cholesterol derivatives can further affect delivery efficacy and biodistribution of lipid nanoparticles. For example, cholesterol analogues with C-24 alkyl phytosterols increase the in vivo delivery efficacy of lipid nanoparticle-mRNA formulations ${ }^{123}$. Here, the length of the hydrophobic tails of the cholesterol analogues, the flexibility of sterol rings and the polarity of 
Cationic lipids

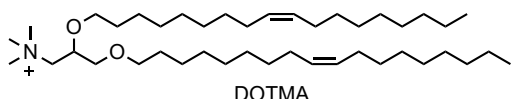

$$
\begin{aligned}
& \underbrace{N_{O}^{O}}_{\text {DOTAP }}
\end{aligned}
$$

Ionizable lipids

$$
\text { (c) }
$$

(c)

Lipid H (SM-102)

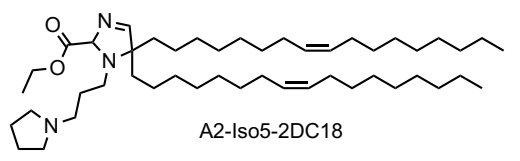

$$
\text { (N) }
$$

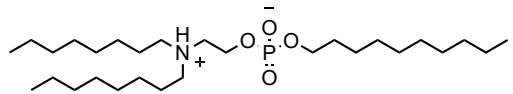

$$
\text { 9A1P9 }
$$

\section{Other types of lipids}

$$
\text { (N) }
$$

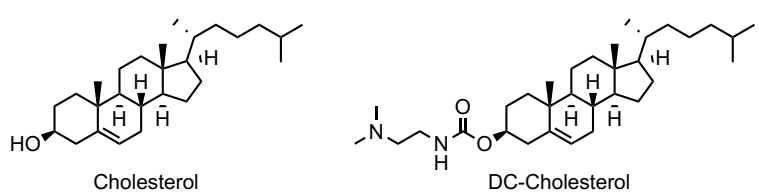

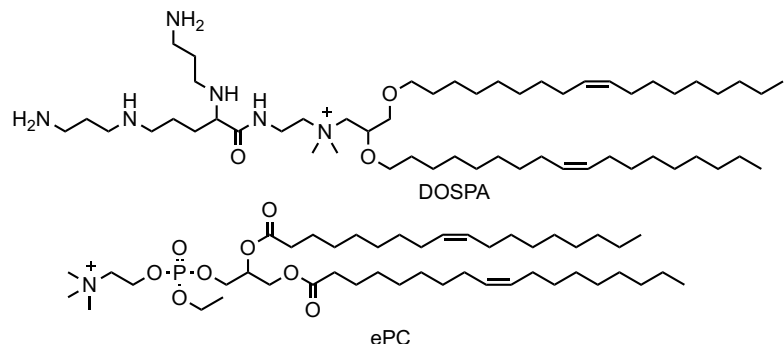
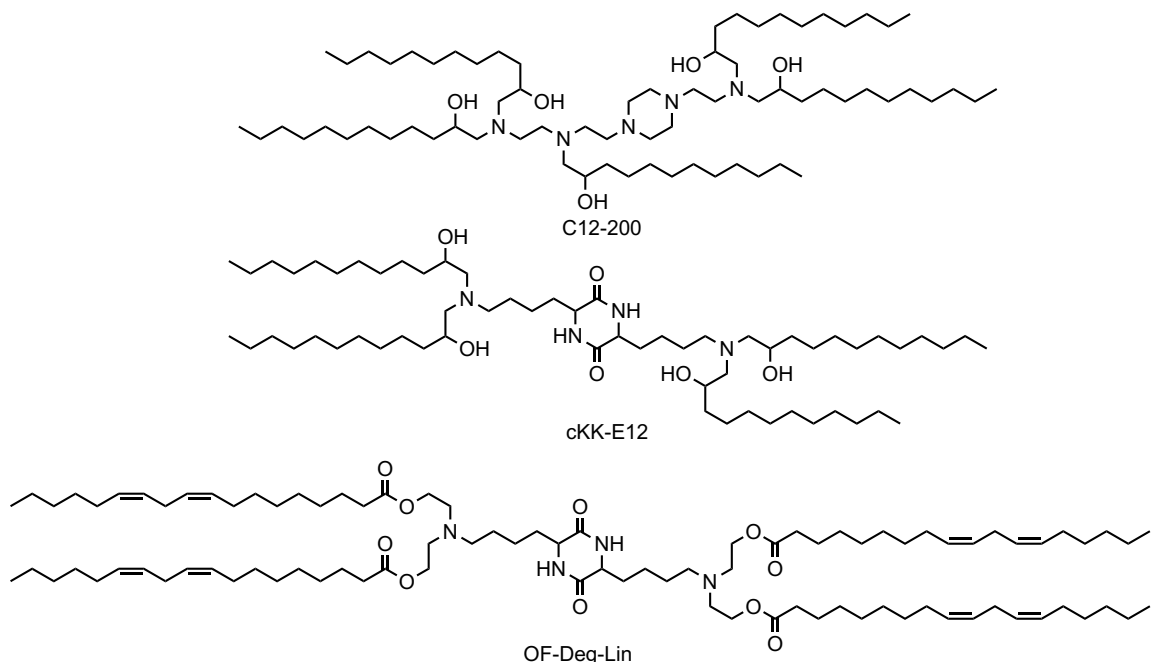<smiles>CC(C)CCCCCCCCOC(=O)CCN(CCCN(C)CCCN(CCC(=O)OCCCCCCCC(C)C)CCC(=O)OCCCCCCCC(C)C)CCC(=O)OCCCCCCCC(C)C</smiles>
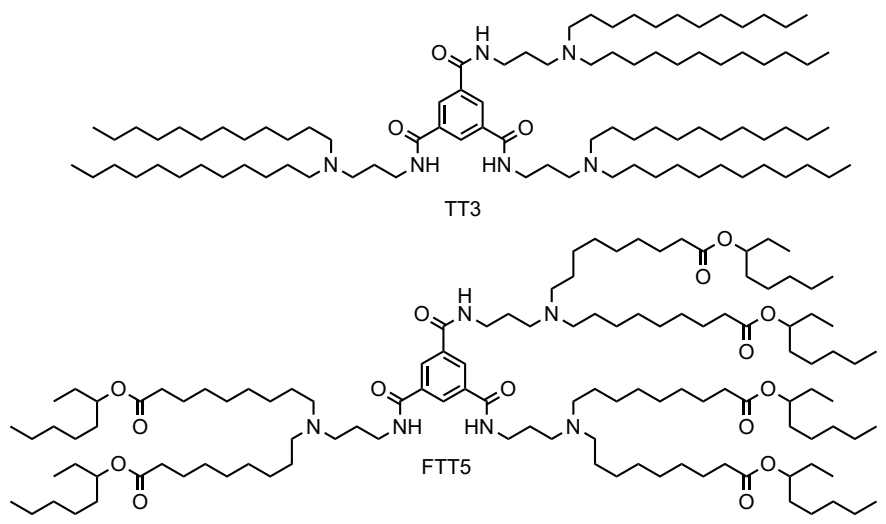
4 Fig. 2 | Chemical structures of lipids and lipid derivatives used for mRNA delivery.

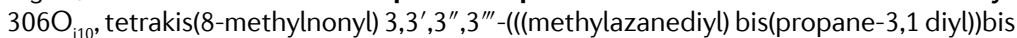
(azanetriyl))tetrapropionate; 9A1P9, decyl (2-(dioctylammonio)ethyl) phosphate; A2-Iso5-2DC18, ethyl 5,5-di((Z)-heptadec-8-en-1-yl)-1-(3-(pyrrolidin-1-yl)propyl)-2, 5-dihydro-1H-imidazole-2-carboxylate; ALC-0315, ((4-hydroxybutyl)azanediyl) bis(hexane-6,1-diyl)bis(2-hexyldecanoate); ALC-0159, 2-[(polyethylene glycol)-2000]$\mathrm{N}, \mathrm{N}$-ditetradecylacetamide; $\beta$-sitosterol, $(3 \mathrm{~S}, 8 \mathrm{~S}, 9 \mathrm{~S}, 10 R, 13 R, 14 \mathrm{~S}, 17 R)-17-((2 R, 5 R)$ 5-ethyl-6-methylheptan-2-yl)-10,13-dimethyl-2,3,4,7,8,9,10,11,12,13,14,15,16,17tetradecahydro-1H-cyclopenta[a]phenanthren-3-ol; BAME-O16B, bis(2-(dodecyldisulfanyl)ethyl) 3,3'-((3-methyl-9-oxo-10-oxa-13,14-dithia-3,6-diazahexacosyl)azanediyl) dipropionate; BHEM-Cholesterol, 2-((((3S,8S,9S,10R,13R,14S,17R)-10,13-dimethyl-17((R)-6-methylheptan-2-yl)-2,3,4,7,8,9,10,11,12,13,14,15,16,17-tetradecahydro- $1 \mathrm{H}$ cyclopenta[a]phenanthren-3-yl)oxy)carbonyl)amino)-N,N-bis(2-hydroxyethyl)- $\mathrm{N}$ methylethan-1-aminium bromide; C12-200, 1,1'-((2-(4-(2-((2-(bis(2-hydroxydodecyl) amino)ethyl) (2-hydroxydodecyl)amino)ethyl) piperazin-1-yl)ethyl)azanediyl) bis(dodecan2-ol); cKK-E12, 3,6-bis(4-(bis(2-hydroxydodecyl)amino)butyl)piperazine-2,5-dione; DC-Cholesterol, $3 \beta-\left[N-\left(N^{\prime}, N^{\prime}\right.\right.$-dimethylaminoethane)-carbamoyl]cholesterol; DLin-MC3DMA, (6Z,9Z,28Z,31Z)-heptatriaconta-6,9,28,31-tetraen-19-yl 4-(dimethylamino) butanoate; DOPE, 1,2-dioleoyl-sn-glycero-3-phosphoethanolamine; DOSPA, 2,3dioleyloxy-N-[2-(sperminecarboxamido)ethyl]-N,N-dimethyl-1-propanaminium trifluoroacetate; DOTAP, 1,2-dioleoyl-3-trimethylammonium-propane; DOTMA, 1,2-di-O-octadecenyl-3-trimethylammonium-propane; DSPC, 1,2-distearoyl-snglycero-3-phosphocholine; ePC, ethylphosphatidylcholine; FTT5, hexa(octan-3-yl) 9,9',9", $9^{\prime \prime \prime}, 9^{\prime \prime \prime}, 9^{\prime \prime \prime \prime}$ - ((((benzene-1,3,5-tricarbonyl)yris(azanediyl)) tris (propane-3,1-diyl)) tris(azanetriyl))hexanonanoate; Lipid H (SM-102), heptadecan-9-yl 8-((2-hydroxyethyl) (6-oxo-6- (undecyloxy)hexyl)amino) octanoate; OF-Deg-Lin, (((3,6-dioxopiperazine-2, 5-diyl)bis(butane-4, 1-diyl))bis(azanetriyl))tetrakis(ethane-2,1-diyl) (9Z,9'Z,9"Z,9'"Z, 12Z,12'Z,12"Z,12'"Z)-tetrakis (octadeca-9,12-dienoate); PEG2000-DMG, 1,2-dimyristoylrac-glycero-3-methoxypolyethylene glycol-2000; $\Pi 33, N^{1}, N^{3}, N^{5}$-tris(3-(didodecylamino) propyl)benzene-1,3,5-tricarboxamide.

hydroxy groups impact delivery efficacy ${ }^{123}$. In addition, lipid nanoparticles formulated with cholesterol derivatives adopt a polyhedral shape, and not a spherical shape, with multilamellarity and lipid partitioning ${ }^{124}$. Lipid nanoparticles containing cholesteryl oleate further show higher selectivity for liver endothelial cells than for hepatocytes ${ }^{125}$. Moreover, oxidative modifications on the cholesterol tail enable lipid nanoparticles to accumulate more in liver endothelial cells and Kupffer cells than in hepatocytes ${ }^{126}$.

PEG-lipids can have multiple effects on the properties of lipid nanoparticles ${ }^{14,17,72,127-129}$. The amount of PEGlipids can affect particle size and zeta potential ${ }^{17,72}$. PEG-lipids can further contribute to particle stability by decreasing particle aggregation ${ }^{14,17,127}$, and certain PEG modifications prolong the blood circulation time of nanoparticles by reducing clearance mediated by the kidneys and the mononuclear phagocyte system (MPS) ${ }^{14,17,127-129}$. Finally, PEG-lipids can be used to conjugate specific ligands to the particle for targeted delivery. The extent of these effects depend on the proportions and properties of the PEG-lipids (such as PEG molar mass and lipid length $)^{17,72,127-129}$. For example, 1,2-dimyristoyl-rac-glycero-3-methoxypolyethylene glycol-2000 ( $\mathrm{PEG}_{2000}$-DMG) and 1,2-distearoylrac-glycero-3-methoxypolyethylene glycol-2000 $\left(\mathrm{PEG}_{2000}-\mathrm{DSG}\right)$ are neutral PEG-lipids, and the length of their saturated alkyl chains is $\mathrm{C} 14$ and $\mathrm{C} 18$, respectively ${ }^{129}$. Lipid nanoparticle-siRNA formulations containing $\mathrm{PEG}_{2000}$-DMG have shorter circulation times and higher delivery efficacy in vivo than formulations containing $\mathrm{PEG}_{2000}-\mathrm{DSG}^{129}$. This difference may be attributed to the faster dissociation of
$\mathrm{PEG}_{2000}$-DMG from lipid nanoparticles, compared with $\mathrm{PEG}_{2000}$-DSG, which may benefit cellular uptake and endosomal escape of lipid nanoparticles ${ }^{128,129}$.

\section{Overcoming physiological barriers}

To function in vivo, lipid nanoparticle-mRNA formulations need to overcome multiple extracellular and intracellular barriers ${ }^{7,11}$ (FIG. 3a). First, mRNA needs to be protected from nuclease degradation in physiological fluids ${ }^{7,11}$. Second, the formulation should evade the interception by the MPS and clearance by renal glomerular filtration post systemic administration ${ }^{7,11}$. Third, lipid nanoparticle-mRNA systems need to reach target tissues, followed by internalization by target cells ${ }^{7,11}$. Finally, mRNA molecules must escape endosomes to reach the cytoplasm, where translation occurs ${ }^{7,11}$.

Lipid nanoparticle-mRNA formulations manufactured by rapid mixing exhibit a stable nanostructure ${ }^{17,130,131}$, in which mRNA molecules can be encapsulated in the interior core through electrostatic interactions with the $\operatorname{lipids}^{17,131}$. This structural feature protects mRNA molecules from nuclease degradation and increases nanoparticle stability in physiological fluids ${ }^{17,46}$. Incorporating PEG-lipids further decreases recognition by the MPS and clearance by renal filtration ${ }^{17,127}$. Additionally, targeted biodistribution of lipid nanoparticle-mRNA formulations can be improved by further modifying and optimizing the nanoparticle $26,27,69,114,132-135$; for example, nanoparticles can be coated with antibodies ${ }^{132}$ to deliver mRNA molecules into inflammatory leukocytes and epidermal growth factor receptor (EGFR)-positive tumour cells for treating inflammatory bowel disease ${ }^{69}$ and cancer ${ }^{133}$, respectively. Organ selectivity can also be achieved by adjusting the proportions of lipid components, for example, to design spleen-targeted mRNA vaccines $^{26,27}$ or lung-targeted genome editing delivery systems $^{114,134}$.

Once they reach target cells, lipid nanoparticles can be internalized by multiple mechanisms, including macropinocytosis and clathrin-mediated and caveolae-mediated endocytosis ${ }^{10,17}$. The endocytic pathway depends on the properties of the nanoparticle and the cell type ${ }^{100,108,136}$. Following cellular internalization, lipid nanoparticles are usually trapped in endosomal compartments ${ }^{137-139}$. Indeed, only a small amount of lipid nanoparticles may be able to escape from the endosome ${ }^{137-139}$. Thus, endosomal escape is crucial for effective mRNA delivery. Although the mechanism has not yet been fully understood, positively charged lipids may facilitate electrostatic interaction and fusion with negatively charged endosomal membranes, resulting in the leak of mRNA molecules into the cytoplasm ${ }^{7,11,14,17,100}$. Endosomal escape can be increased by optimizing the pKa values of ionizable lipids ${ }^{66,76-78,88,100,140}$. Furthermore, the properties of lipidic tails can affect endosomal escape of lipid nanoparticles ${ }^{64,97,114,141}$; for example, some lipids with branched tails show enhanced endosomal escape compared with their counterparts with linear tails, owing to stronger protonation at endosomal $\mathrm{pH}$ (REF. ${ }^{97}$ ). In addition, modulating the type (for example, DSPC and DOPE) and ratio of lipids may improve endosomal escape $\mathrm{e}^{90,104,116,123,136,142}$. 

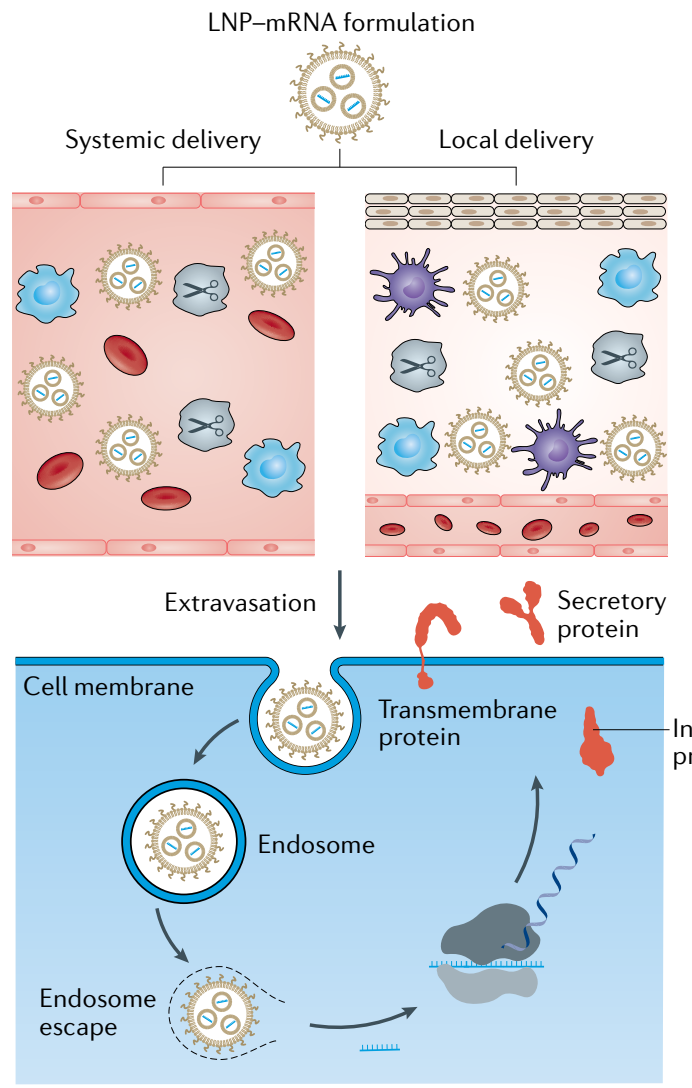

b

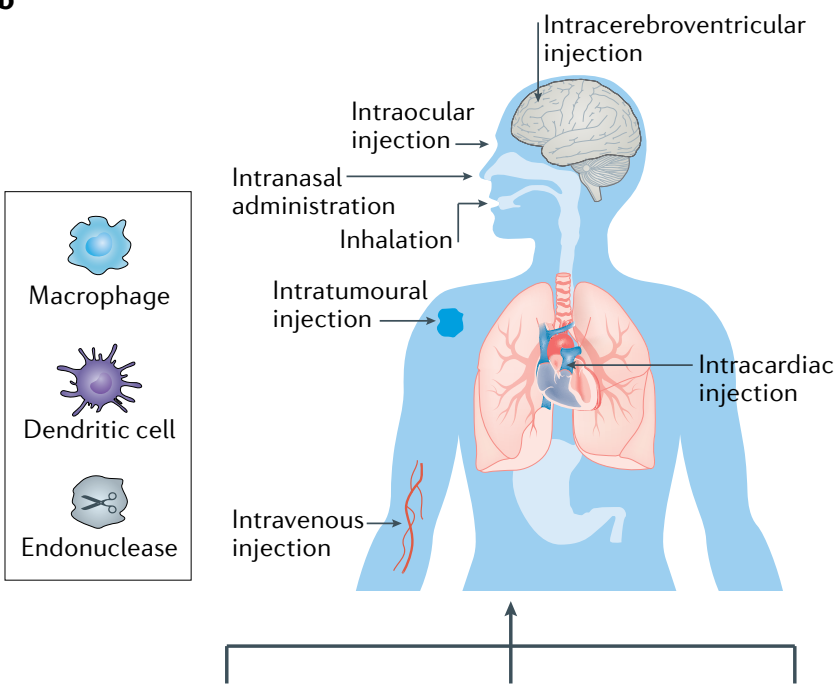

Fig. 3 | Delivery barriers and administration routes for lipid nanoparticle-mRNA formulations. a | Physiological barriers for lipid nanoparticle-mRNA (LNP-mRNA) formulations post systemic and local delivery. $\mathbf{b} \mid$ Administration routes for LNP-mRNA formulations. Panel b reprinted from REF. ${ }^{155}$, Springer Nature Limited.

\section{Administration routes}

Administration routes can greatly influence organ distribution, expression kinetics and therapeutic outcomes of lipid nanoparticle-mRNA formulations ${ }^{143,144}$. The administration route is often determined by the properties of the nanoparticles and therapeutic indications (FIG. 3b). After intravenous (i.v.) administration, many lipid nanoparticles can accumulate in the liver. The liver is inherently capable of producing secretory proteins and, therefore, i.v. administration of lipid nanoparticlemRNA formulations can be used to produce proteins that are missing in inherited metabolic and haematological disorders, or to produce antibodies to neutralize pathogens or target cancer cells ${ }^{39,95,145-147}$. These applications require protein translation without stimulation of an immune response, which may limit the efficiency of repeated dosing. However, i.v. administration may also lead to accumulation of lipid nanoparticles in multiple lymph nodes throughout the body, which could increase immune responses to mRNA vaccines. For example, i.v. administration of mRNA vaccines has been shown to induce stronger antigen-specific cytotoxic $\mathrm{T}$ cell responses compared with local injection ${ }^{26,148,149}$. Broad distribution of mRNA vaccines in the body may lead to systemic adverse effects, and, thus, it may be necessary to develop lipid nanoparticles that allow targeted delivery of mRNA vaccines into tissues with abundant immune cells ${ }^{26,150}$.
Topical administration routes have also been explored for mRNA therapeutics. Topical administration aims at achieving local therapeutic effects; for example, local injection of lipid nanoparticle-mRNA formulations enables supplementation of therapeutic proteins in specific tissues, such as heart ${ }^{49,93}$, eyes ${ }^{56,72,151}$ and brain $^{67,152}$. Of note, administration of mRNA encoding vascular endothelial growth factor (VEGF) has been shown to lead to functional protein expression in the skin even in the absence of lipid nanoparticles ${ }^{153}$. Indeed, mRNA delivery by direct injection into the heart muscle of patients undergoing coronary bypass surgery is currently being tested in a randomized phase II trial (NCT03370887) ${ }^{154}$. Moreover, lipid nanoparticlemRNA formulations can be administered into the lungs by inhalation $^{71}$, for example, MRT5005 (NCT03375047).

Local administration can also prime systemic responses; for example, intradermal (i.d.), intramuscular (i.m.) and subcutaneous (s.c.) injection are commonly used for vaccination ${ }^{17,155}$, because resident and recruited antigen-presenting cells (APCs) are present in the skin and muscle, which can internalize and process mRNA-encoded antigens ${ }^{17,155}$. Furthermore, the vascular and lymphatic vessels of these tissues help APCs and mRNA vaccines to centre the draining lymph nodes to stimulate $\mathrm{T}$ cell immunity ${ }^{17,155}$. Indeed, both i.m. and i.d. administration of lipid nanoparticle-mRNA vaccines produce robust immune responses at a well-tolerated 
dose in human trials ${ }^{156,157}$. Vaccination can also be done by intranasal administration, because APCs in the peripheral lymph nodes can readily endocytose administered lipid nanoparticle-mRNA formulations ${ }^{17,34,155,158-160}$. In addition, lipid nanoparticle-mRNA formulations encoding immune stimulators can be directly delivered into tumour tissue by intratumoural injection ${ }^{106,161-163}$, to boost a local pro-inflammatory environment, which leads to immune cell activation and subsequent priming of systemic anticancer responses ${ }^{106,161-163}$. Finally, in utero administration of lipid nanoparticle-mRNA formulations can be applied to deliver mRNA to mouse fetuses ${ }^{164}$, achieving protein expression in fetal livers, lungs and intestines ${ }^{164}$.

\section{Considerations for clinical translation}

The properties of lipid nanoparticle-mRNA formulations need to be carefully characterized and considered for the desired application. Lipid nanoparticle-mRNA formulations may need different properties as vaccines than as therapeutics to achieve optimal therapeutic effects, for example, distinct biodistribution profiles. Vaccines need to interact with immune cells, whereas therapeutics are targeted to specific organs. Therefore, lipid nanoparticle-mRNA formulations should be designed according to biomedical demand. To translate lipid nanoparticle-mRNA systems from bench to bedside, good manufacturing practice (GMP) is crucial to ensure drug quality and therapeutic effects, in addition to considerations such as storage conditions and safety profiles.

Good manufacturing practice. The preparation of a linearized DNA template is the initial step of GMP production of $\mathrm{mRNA}^{2,4,165,166}$. Based on the DNA template, the mRNA is then transcribed in vitro in the presence of an RNA polymerase and ribonucleoside triphosphates $2,4,165,166$. The residual DNA template is

\section{Box 1 | Engineering mRNA molecules}

mRNA normally contains five structural elements, that is, a $5^{\prime}$ cap, a $3^{\prime}$ poly(A) tail, a protein-coding sequence and $5^{\prime}$ and $3^{\prime}$ untranslated regions (UTRs) ${ }^{2,4,7,13}$. These elements are crucial for initiation, translation, termination, post-transcriptional modification and decay of mRNA molecules ${ }^{2,4,7,13}$. These elements can be engineered to improve the stability and translational efficiency of mRNA ${ }^{2,4,7,13}$.

- Incorporation of 5' cap analogues allows initiation of the translation complex with the eukaryotic translation initiation factor $4 \mathrm{E}$. Such $5^{\prime}$ cap analogues may be more resistant to decapping enzymes

- The $3^{\prime}$ poly(A) tail is involved in interactions with the poly(A)-binding protein. Optimization of the length of the poly $(\mathrm{A})$ tail and its composition can stabilize the mRNA and increase protein expression

- UTRs interact with multiple RNA-binding proteins and microRNAs, and, thus, sequence engineering of $5^{\prime}$ and $3^{\prime}$ UTRs can increase the half-life and translational efficiency of mRNA

- Codon optimization (for example, replacing rare codons with synonymous frequent codons) can accelerate the translation rate. Codon optimization can also form favourable secondary structures, improving translational efficiency

- Incorporating chemically modified nucleosides can decrease immunogenicity and increase translation of mRNA

- Circular RNA (circRNA) design can extend the duration of mRNA translation because circRNA is resistant to nuclease-mediated degradation removed by DNase digestion and the mRNA molecules are capped by chemical or enzymatic methods ${ }^{2,4,165,166}$. Finally, mRNA is purified by microbeads-based precipitation or chromatographic methods to remove the enzymes, free nucleotides, truncated nucleic acid fragments and double-stranded RNA ${ }^{2,4,165,166}$. The purified mRNA can be dissolved in a storage buffer, filtered for sterilization and frozen for long-term storage $\mathrm{e}^{2,4,165,166}$. To increase the stability and translational efficiency of mRNA, various approaches have been explored to optimize its structural elements (BOX 1).

Historically, lipid nanoparticle-nucleic acid formulations were produced by thin-film hydration, reverse-phase evaporation and other methods ${ }^{167}$. The sizes of lipid nanoparticles were further homogenized by extrusion techniques ${ }^{167}$. Lipid nanoparticle-mRNA formulations are now commonly manufactured by rapid mixing; here, an ethanol phase (lipid components) and an aqueous phase (mRNA molecules) are mixed under specific conditions (that is, $\mathrm{pH}$ and flow rate) ${ }^{17,131}$. This technique allows reproducible and scalable production of lipid nanoparticle-mRNA formulations that show high encapsulation efficiency and homogeneous size distribution ${ }^{17,131}$. mRNA is susceptible to degradation and, thus, formulation buffers should be free of any ribonuclease contaminations ${ }^{2,4}$. Lipid nanoparticle-mRNA formulations are further purified to remove organic solvents and residual components, and the final mRNA concentration can be further increased by enrichment. The filtered and frozen lipid nanoparticle-mRNA formulations are then subject to a series of GMP standard tests, including evaluation of physical parameters (such as mRNA encapsulation, particle sizes, charges), compendial testing (such as sterility, bacterial endotoxins, particulate matter, osmolality) and other quality testing.

Stability and storage. Storage conditions of lipid nanoparticle-mRNA formulations are an important consideration for their clinical translation, because storage (aqueous, freezing and lyophilized storage) and the type of cryoprotectants (sucrose, trehalose or mannitol) affect the long-term stability of lipid nanoparticle-mRNA formulations $\mathrm{s}^{168}$. For example, the addition of $5 \%(\mathrm{w} / \mathrm{v})$ sucrose or trehalose to lipid nanoparticle-mRNA formulations, stored in liquid nitrogen, allows maintenance of mRNA delivery efficacy for at least 3 months in vivo ${ }^{168}$. Of note, the authorized COVID-19 mRNA vaccines are both stored in freezing conditions in the presence of sucrose $\mathrm{e}^{17}$. mRNA- 1273 is stored at $-15^{\circ} \mathrm{C}$ to $-20^{\circ} \mathrm{C}$ and is directly injected after thawing ${ }^{17}$, whereas BNT162b2 is stored at $-60^{\circ} \mathrm{C}$ to $-80^{\circ} \mathrm{C}$ and requires thawing and dilution by saline before injection ${ }^{17}$. Recently, the European Medicines Agency (EMA) has approved storage of BNT $162 \mathrm{~b} 2$ at $-15^{\circ} \mathrm{C}$ to $-25^{\circ} \mathrm{C}$ for 2 weeks based on new stability data. Although cold-chain transportation can maintain vaccine activity, the development of lipid nanoparticle-mRNA formulations that do not require cold or frozen storage would not only decrease production and transportation costs but also expedite the process of vaccination. Therefore, it is important to investigate the factors impacting long-term storage of lipid nanoparticle-mRNA formulations. 
Safety profiles. The safety profile of lipid nanoparticlemRNA formulations correlates with the lipid components and mRNA molecules. Lipid components may activate host immune responses following systemic or local administration; for example, PEG-lipids could induce hypersensitivity reactions by stimulating the complement system ${ }^{127,169}$. Moreover, anti-PEG antibodies could result in fast systemic clearance of subsequently administered PEGylated nanoparticles by accelerated blood clearance ${ }^{127,169}$. The accelerated blood clearance phenomenon may change the bioavailability and biodistribution of the drug encapsulated in PEGylated nanoparticles and, thus, cause side effects ${ }^{127,169}$. To ameliorate safety concerns, numerous natural and synthetic polymers have been investigated as alternatives to PEG, of which several are under evaluation in clinical trials ${ }^{127,169}$. Cationic and ionizable lipids have also been reported to stimulate the secretion of pro-inflammatory cytokines and reactive oxygen species ${ }^{170-173}$. Although the immunogenicity of these lipids has not yet been fully understood, complement system and Toll-like receptors may participate in innate immune activation ${ }^{170,173-175}$. Cytotoxicity of lipid materials is also a safety concern, depending on the dose, lipid properties and cell types ${ }^{176,177}$. In vivo application of lipid nanoparticles has been reported to induce liver and lung injuries in rodents ${ }^{170,173}$, which may be attributed to the cytotoxicity of the materials and the induction of pro-inflammatory factors ${ }^{171,178}$. To improve the biocompatibility of lipid nanoparticles, biodegradable lipids can be applied ${ }^{76-78,108,179}$.

The immunogenicity of IVT mRNA is another safety concern, although eliciting cellular and humoral immunity may be advantageous for vaccination. Nevertheless, immune responses to IVT mRNA may also suppress antigen expression and negatively affect vaccine efficacy ${ }^{175,180,181}$. Moreover, immune activation is undesirable for some mRNA applications, such as protein replacement therapies and genome editing. To minimize the immunogenicity of mRNA, two approaches are commonly used. Chemical modifications of specific IVT mRNA nucleotides, such as pseudouridine $(\psi)$ and $N^{1}$-methylpseudouridine $(\mathrm{m} 1 \psi)$, can reduce innate immune sensing of exogenous mRNA translation ${ }^{2,4,7,182}$. Chromatographic purification can remove doublestranded RNA, an analogue of viral genome, in IVT mRNA preparations, diminishing immune activation and increasing translational efficiency ${ }^{2,47,183}$. The IVT mRNA molecules used in the mRNA-1273 and BNT162b2 COVID-19 vaccines were prepared by replacing uridine with $\mathrm{m} 1 \psi^{17,19,21}$, and their sequences were optimized to encode a stabilized pre-fusion spike protein with two pivotal proline substitutions ${ }^{17,19,21}$.

\section{Preclinical studies and clinical trials}

The features of lipid nanoparticle-mRNA formulations have been thoroughly preclinically and clinically investigated, which has allowed the rapid development and clinical use of the COVID-19 lipid nanoparticlemRNA vaccines. For example, the clinical-grade COVID-19 vaccine, mRNA-1273, was produced within a month after the SARS-CoV-2 genome sequence was available ${ }^{15,17}$. About 2, 5 and 6 months from sequence availability, clinical trial phases I, II and III were initiated, respectively ${ }^{15,17}$. Finally, mRNA-1273 obtained Emergency Use Authorization from the FDA and conditional marketing authorization from the EMA within a year ${ }^{15,17}$. Many other lipid nanoparticle-mRNA formulations are in clinical trials for the treatment of infectious diseases, cancer and genetic disorders ${ }^{2,4,8,12-14}$ (TABLES 1,2). Moreover, mRNA-based cellular reprogramming, tissue regeneration and genome editing have shown therapeutic potential in preclinical studies ${ }^{2,4,8,12-14}$.

Infectious diseases. Vaccines are the most effective approach to control and eradicate epidemics. The first mRNA vaccine was made of liposomes and mRNA encoding an influenza virus nucleoprotein ${ }^{184}$. This vaccine, designed in 1993, was able to induce virus-specific cytotoxic $\mathrm{T}$ cell responses in mice ${ }^{184}$. Since then, lipid nanoparticle-mRNA formulations have emerged as a potent alternative to conventional vaccine platforms, owing to their unique features ${ }^{4,15,17}$. First, mRNA is a non-infective and non-integrating agent with the ability to encode a broad range of antigens ${ }^{4,15,17}$. Second, mRNA vaccines can combine different antigen mRNAs; for example, six separate mRNAs have been incorporated in a cytomegalovirus vaccine, five of which encode a single pentameric antigen and one encodes a glycoprotein antigen ${ }^{185}$. Finally, GMP-grade lipid nanoparticle-mRNA vaccines can be manufactured for specific antigens in a short period of time, compared with other vaccine platforms, such as recombinant proteins and inactivated vaccines $^{2,4,15,17}$. These features make lipid nanoparticlemRNA formulations a flexible and on-demand vaccine platform to rapidly respond to emerging infectious pathogens ${ }^{4,15,17}$. However, the instability and short halflife of mRNA need to be carefully considered. In addition, safety concerns and storage conditions of lipid nanoparticles need to be determined before clinical use.

To address these concerns, the engineering of mRNA molecules (BOX 1) and the design of lipid nanoparticles have been optimized ${ }^{2,4,7-17}$, which has contributed to the rapid development and clinical assessment ${ }^{15,17}$ of the two COVID-19 mRNA vaccines, mRNA-1273 $(\text { NCT04470427) })^{19,20}$ and BNT162b2 (NCT04368728) $)^{21}$. Both vaccines use ionizable lipid nanoparticles to deliver nucleoside-modified mRNA encoding the full-length spike protein of SARS-CoV-2 (FIG. 4). Applying a primeboost vaccination method, the vaccines induce high levels of antigen-specific antibodies and elicit robust $\mathrm{T}$ helper 1 cell responses ${ }^{19-21}$. Moreover, the vaccines showed similar efficacy ( $95 \%)$ in phase III clinical trials $^{19-21}$. Other COVID-19 vaccines based on lipid nanoparticle-mRNA formulations are also under evaluation in different clinical phases (TABLE 1). In preclinical studies, some vaccine candidates showed protective effects by delivering self-amplifying RNA encoding the spike protein $^{40,186,187}$ (BOX 2), a cocktail of mRNAs encoding three viral proteins ${ }^{74}$, modified mRNA encoding the receptor-binding domain ${ }^{188}$ or spike mRNA with engineered untranslated regions ${ }^{107}$.

Lipid nanoparticle-mRNA vaccines are also being investigated as influenza vaccines ${ }^{29,41,156,157,159,184,189-191}$, with some formulations in clinical trials (TABLE 1). 


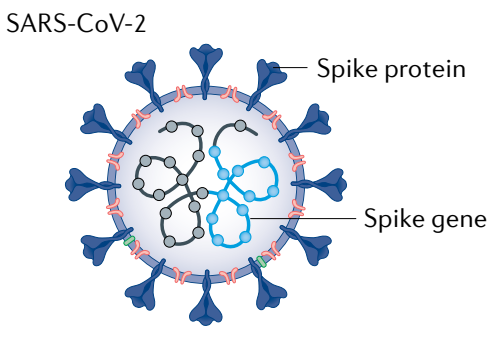

LNP-mRNA vaccine

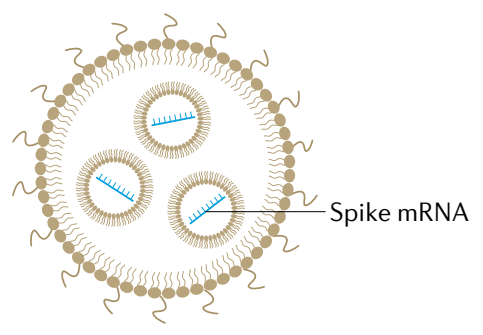

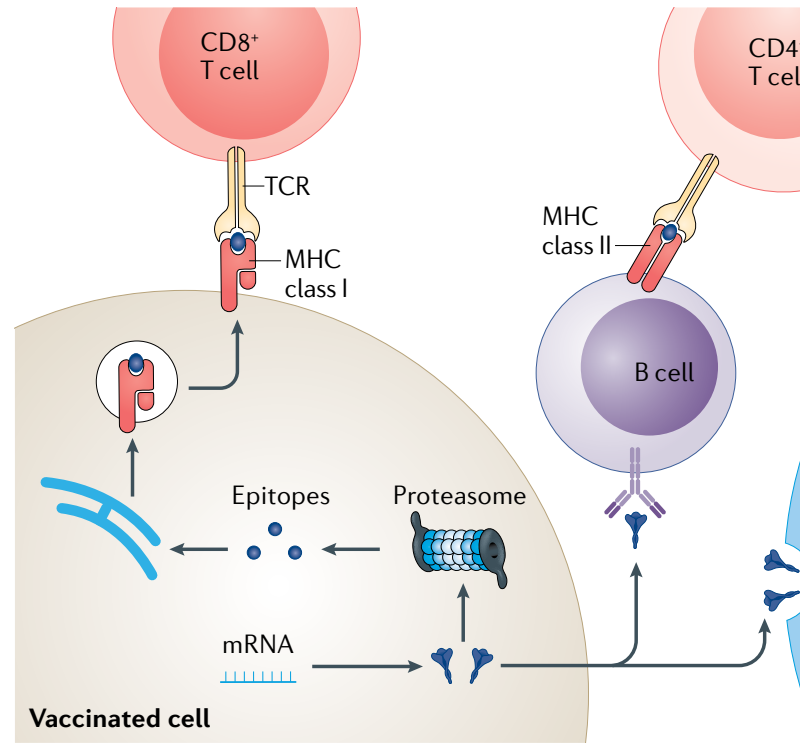

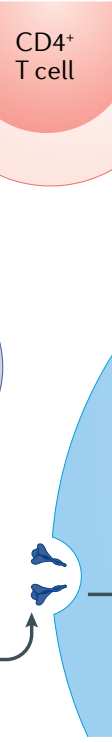

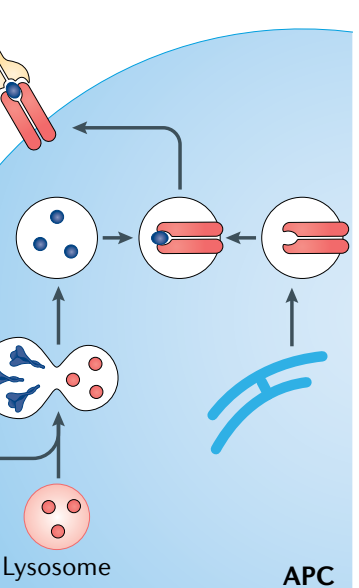

Fig. 4 | Lipid nanoparticle-mRNA formulations as COVID-19 vaccines. After intramuscular injection, lipid nanoparticle-mRNA (LNP-mRNA) vaccines are internalized by somatic cells (for example, muscle cells) and tissueresident or recruited antigen-presenting cells (APCs) ${ }^{2,47,11,17}$. Moreover, LNP-mRNA vaccines can centre draining lymph nodes, where various immune cells reside, including naive $T$ and $B$ cells ${ }^{2,4,7,11,17}$. Spike antigens expressed in the cytoplasm are degraded by proteasomes ${ }^{2,4,7,11,17}$ and major histocompatibility complex (MHC) class I presents the resultant epitopes to $\mathrm{CD}^{+} \mathrm{T}$ cells $\mathrm{s}^{2,4,7,11,17}$. Spike antigens can also be endocytosed by APCs. These antigens are degraded in the lysosomes of APCs and presented by MHC II molecules for $\mathrm{CD} 4^{+} \mathrm{T}$ cells $\mathrm{s}^{2,4,7,11,17}$. In addition, secreted spike antigens can be internalized by $B$ cell receptors and processed for presentation to $C D 4^{+} T$ cells by MHC class II molecules ${ }^{24,7,11,17}$. COVID-19, coronavirus disease 2019; SARSCoV-2, severe acute respiratory syndrome coronavirus 2; TCR, T cell receptor.
Haemagglutinin is an essential surface antigen of influenza viruses ${ }^{156,157}$. The mRNA-1440 and mRNA-1851 vaccines, which are composed of lipid nanoparticles and mRNA encoding haemagglutinin from H10N8 and H7N9 influenza viruses, respectively, have completed phase I clinical studies (NCT03076385 and NCT03345043 $)^{156,157}$. After i.m. prime-boost vaccination, humoral immune responses were evaluated by haemagglutination inhibition (HAI) and microneutralization $(\mathrm{MN})$ assays. A 100- $\mu$ g dose level induced $78.3 \%$ (HAI) and $87.0 \%(\mathrm{MN})$ seroconversion for H10N8 $\left(\right.$ REFS $^{156,157}$ ) and a $50-\mu \mathrm{g}$ dose level resulted in 96.3\% (HAI) and 100\% (MN) seroconversion for H7N9 $\left(\mathrm{REFS}^{156,157}\right)$. Lipid nanoparticle-mRNA formulations are further being explored for Zika virus vaccines ${ }^{38,190,192-194}$. The vaccine mRNA-1893 is a clinical candidate that contains lipid nanoparticles with mRNA encoding Zika virus premembrane and envelope (prM-E) proteins ${ }^{193,194}$. According to $\mathrm{mRNA}-1893$ interim phase I data (NCT04064905) reported by Moderna, $10-\mu \mathrm{g}$ and $30-\mu \mathrm{g}$ dose levels (prime-boost regimen) induced $94 \%$ and $100 \%$ seroconversion, respectively, and both dose levels were generally well tolerated.

A series of clinical trials of mRNA vaccines have been initiated against human metapneumovirus, cytomegalovirus, respiratory syncytial virus, rabies virus and chikungunya virus (TABLE 1). Furthermore, lipid nanoparticle-mRNA vaccines have been tested against other viruses in animal models, including human immunodeficiency virus ${ }^{28,144,181,190}$, Powassan virus ${ }^{195}$, Venezuelan equine encephalitis virus ${ }^{196}$, dengue virus ${ }^{73}$ and Ebola virus ${ }^{197}$.

Apart from viral infections, lipid nanoparticlemRNA vaccines have been reported to induce immune protection against parasitical ${ }^{31}$ and bacterial ${ }^{30,198}$ infections. In addition, lipid nanoparticle-mRNA formulations have been applied to produce therapeutic proteins or antibodies ${ }^{39,75,145,146}$, to edit virus genomes ${ }^{105}$ and to engineer immune cells ${ }^{136}$. For example, a vitamin C-derived lipid nanoparticle allows mRNA delivery into primary macrophages ${ }^{136}$. Adoptive transfer of macrophages engineered by delivering mRNA that encodes an antimicrobial peptide considerably reduced bacterial burden and increased survival in mice with multidrug-resistant bacterial sepsis ${ }^{136}$. Furthermore, the lipid nanoparticle-mRNA formulation mRNA-1944 (NCT03829384), designed to generate anti-chikungunya virus antibody (CHKV-IgG) in vivo, is under clinical evaluation. A single i.v. injection of $0.1,0.3$ and $0.6 \mathrm{mg} \mathrm{kg}^{-1}$ lipid nanoparticle-mRNA formulation produced 2.0, 7.9 and $10.2 \mu \mathrm{g} \mathrm{ml}^{-1} \mathrm{CHKV-IgG} 24 \mathrm{~h}$ post injection, respectively. The half-life of CHKV-IgG was about 69 days and the dose levels were reasonably well tolerated, including $0.3 \mathrm{mg} \mathrm{kg}^{-1}$ given twice a week apart.

Cancer. The attempt of mRNA-based cancer immunotherapies dates back to 1995, when i.m. injection of mRNA encoding carcinoembryonic antigens was shown to induce antigen-specific immune responses in mice ${ }^{199}$. Various cancer vaccines based on lipid nanoparticle-mRNA formulations are currently in clinical trials (TABLE 1). For example, FixVac, which was developed based on the RNA-LPX formulation, is a systemic mRNA vaccine encoding four non-mutated antigens of melanoma ${ }^{200}$. The interim analysis from the phase I trial (NCT02410733) showed that the metabolic activity of the spleen increased post the sixth immunization, 


\section{Box 2 | Self-amplifying RNA and circular RNA}

\section{Self-amplifying RNA $^{2,13}$}

Compared with regular mRNA, self-amplifying RNA (also termed replicon), which was originally derived from an alphavirus genome, has similar basic elements ( $5^{\prime} \mathrm{cap}, 5^{\prime}$ and $3^{\prime}$ untranslated regions, $3^{\prime}$ poly(A) tail) and a long coding region. The coding region contains the sequences for an RNA-dependent RNA polymerase (RDRP), a promoter and structural viral proteins (also known as subgenomic sequence). After delivery into the cytoplasm, self-amplifying RNA (positive-strand mRNA) functions as a translation template for the production of the RDRP. Moreover, the positive-strand mRNA serves as a genomic template for RDRP-mediated replication. The initial replication leads to negative-strand RNA, which acts as template for the generation of the positive-strand viral genome. Meanwhile, the promoter in the negative-strand RNA is recognized by the RDRP, leading to the transcription of capped subgenomic RNA that encodes structural viral proteins. This self-amplification process allows the mass production of virions from limited amounts of virus at infection. Replacing the subgenomic sequence by a gene of interest enables high-level expression of desired proteins. The transient replication generates double-stranded RNA (dsRNA) and, thus, self-amplifying RNA tends to activate innate immune pathways.

\section{Circular RNA ${ }^{13,245,246}$}

Circular RNA (circRNA) is a single-stranded RNA with a closed-loop structure. circRNA does not have a free $5^{\prime}$ cap or $3^{\prime}$ poly(A) tails and is, thus, unsusceptible to degradation by nucleases and more stable than linear RNA. Moreover, circRNA without a stop codon reduces the frequency of ribosome detachment from the RNA, thereby enabling continuous translation and high protein expression. Synthetic circRNA can be made by covalently linking the $3^{\prime}$ and $5^{\prime}$ ends of a linear precursor using enzymatic or chemical methods. Similar to linear mRNA, the chemical modification of specific nucleotides and chromatographic purification can minimize immunogenicity of the RNA and increase translation. Therefore, circularization can improve the stability and half-life of mRNA in a physiological environment. respectively ${ }^{210,211}$. In the monotherapy group, 14 out of 16 patients remained disease-free during the study, with a median follow-up time of 8 months ${ }^{210,211}$. In the combination group, the overall response rate in the cohort (human papillomavirus-negative, immune checkpoint inhibitor-naive, head and neck squamous cell carcinomas) was $50 \%$ and the median progression-free survival was 9.8 months ${ }^{210,211}$. Using a similar lipid nanoparticle formulation, mRNA vaccination has also been shown to elicit specific $\mathrm{T}$ cell responses in patients with gastrointestinal cancer (NCT03480152) ${ }^{212}$.

mRNA vaccines can further be applied to overcome insufficient stimulation of chimeric antigen receptor (CAR) $\mathrm{T}$ cells in the therapy of solid tumours ${ }^{213}$. For example, the RNA-LPX formulation can be used to deliver mRNA encoding claudin 6 (CLDN6), a target for CAR T cell therapy in solid tumours ${ }^{213}$. i.v. injection of this vaccine resulted in CLDN6 expression on splenic dendritic cells and macrophages ${ }^{213}$, promoting the activation of adoptively transferred CLDN6-CAR T cells and leading to suppression of large tumours in mice at a sub-therapeutic CAR T cell dose $\mathrm{e}^{213}$.

Alternatively to vaccination, a pro-inflammatory tumour microenvironment can be induced by lipid nanoparticle-mRNA formulations delivering cytokines or co-stimulatory molecules ${ }^{33,106,161,163,214}$ (TABLE 2). For example, mRNA-2416, a lipid nanoparticle encapsulating mRNA encoding human OX40L (a ligand of OX40), is in clinical evaluation for the treatment of patients with solid tumours (NCT03323398) 214 $^{12}$ In this trial, mRNA2416 was intratumourally administered every 2 weeks for up to 12 doses, with four dose levels from 1 to $8 \mathrm{mg}$ (REF. ${ }^{214}$ ). mRNA-2416 was generally well tolerated at different does levels ${ }^{214}$. Moreover, the injected lesions showed an increase in OX40L expression and enhanced T cell activation ${ }^{214}$. Encouraged by these results, mRNA2752, a lipid nanoparticle formulated with mRNA encoding human OX40L, IL-23 and IL-36 $\gamma$, entered clinical evaluation (NCT03739931) 163,215,216. mRNA-2752 was designed to induce a pro-inflammatory tumour microenvironment and to simultaneously strengthen $\mathrm{T}$ cell expansion, as well as memory responses ${ }^{215,216}$. mRNA-2752 was intratumourally administered every 2 weeks for up to seven doses, alone or in combination with infusion of durvalumab ${ }^{215,216}$. In the 22 patients (monotherapy: $n=15$; combination: $n=7$ ), six had stable disease, one had partial responses with $52 \%$ tumour reduction and five showed tumour shrinkage in treated and/or untreated sites ${ }^{215,216}$. Lipid nanoparticle-mRNA formulations have also been investigated for ex vivo engineering of CAR T cells ${ }^{217}$ and for the production of antibodies, such as anti-CD20 (REF. ${ }^{146}$ ), anti-human epidermal growth factor receptor 2 (HER2) ${ }^{95}$ and anti-CD3/ claudin $6\left(\right.$ REF. $\left.^{147}\right)$. Cancers are often accompanied by mutations in the genome and, therefore, correction of these mutations could be an effective approach for cancer therapies. For example, restoration of the tumour suppressor gene TP53 can induce tumour cell apoptosis and sensitize tumour cells to chemotherapeutics in vivo ${ }^{118,218}$. Similarly, the regulation of other tumour-associated genes, such as PLK1 (REF. ${ }^{133}$ ), Bax ${ }^{219}$, Maspin ${ }^{37}, P_{M} A^{70}$ and $P T E N^{220}$, can delay tumour growth in vivo. 
Genetic disorders. Genetic disorders are caused by inherited or acquired gene mutations, which can cause abnormal protein expression ${ }^{12}$. The supplement of therapeutic proteins can relieve clinical symptoms but can often not provide lasting treatments or cures. Alternatively, gene therapy seeks to modify malfunctioning genetic expression. mRNA-based protein replacement therapies have also emerged as a promising alternative to protein drugs, because mRNA can be translated into desired proteins with in situ post-translational modifications in host cells ${ }^{12}$. Moreover, mRNA can restore different types of protein, including secretory proteins, intracellular proteins and transmembrane proteins ${ }^{12}$.

Clinical trials of protein replacement therapies using lipid nanoparticle-mRNA formulations have mainly focused on inherited metabolic disorders thus far, including ornithine transcarbamylase deficiency (NCT03767270), methylmalonic acidaemia (NCT03810690) and propionic acidaemia (NCT04159103) (TABLE 2). These diseases are characterized by genetic deficiency of key enzymes, leading to an inability to process certain metabolic products ${ }^{12}$. The excessive accumulation of metabolites then results in clinical symptoms and may lead to death ${ }^{12}$. Therefore, the supplement of desired enzymes by lipid nanoparticle-mRNA formulations can slow down disease progression ${ }^{42,221}$. The therapeutic potential of mRNA-based protein replacement therapies has also been tested in other metabolic disorders in preclinical studies, including hereditary tyrosinaemia type I $(\mathrm{HTI})^{142}$, acute intermittent porphyria ${ }^{222,223}$, Fabry disease $^{224,225}$, Crigler-Najjar syndrome type 1 (REF. ${ }^{226}$ ), $\alpha 1$ antitrypsin deficiency ${ }^{227}$, methylmalonic acidaemia/ aciduria $^{228}$, arginase deficiency ${ }^{229}$, citrin deficiency ${ }^{230}$ and glycogen storage disease type I $\left(\mathrm{REF}^{231}\right)$. In addition, mRNA-based protein replacement therapies have been applied to haematological diseases (for example, haemophilia $\mathrm{A}^{232}$, haemophilia $\mathrm{B}^{91,104,233}$ and thrombotic thrombocytopaenic purpura ${ }^{234}$ ), central nervous system disorders $^{67,152}$ (for example, Friedreich's ataxia ${ }^{67}$ ), skin diseases $^{235,236}$ (for example, elastin deficiency ${ }^{235}$ ) and hearing loss ${ }^{237}$ in preclinical studies.

Gene-editing tools further provide the opportunity to correct mutated genes in genetic disorders. Gene-editing components can be delivered by lipid nanoparticle-mRNA formulations to treat genetic diseases, including $\mathrm{HTI}^{92,238}$, hypercholesterolaemia ${ }^{81,96,105,239}$, lipoprotein metabolism disorders ${ }^{85}$ and transthyretin amyloidosis ${ }^{239,240}$, which has been demonstrated in preclinical studies. Intellia Therapeutics further initiated a phase I clinical trial (NCT04601051) to study the safety, pharmacokinetics and pharmacodynamics of NTLA2001 (lipid nanoparticles encapsulating gene-editing components) in patients with hereditary transthyretin amyloidosis.

mRNA-based protein replacement therapies are also in clinical trials for the treatment of cystic fibrosis. Patients with cystic fibrosis usually suffer from repeated airway infections and chronic respiratory problems because of the defective cystic fibrosis transmembrane conductance regulator (CFTR), a chloride channel on epithelial cells ${ }^{12}$. Lipid nanoparticles encapsulating CFTR
mRNA can restore chloride secretion in Cftr-knockout mice $^{68}$. Translate Bio has started a clinical trial (NCT03375047) to evaluate the safety and tolerability of nebulized lipid nanoparticle-mRNA formulations (MRT5005) in patients with cystic fibrosis. In this phase I/II study, patients received a single dose of MRT5005 at three dose levels (8, 16 and 24 mg). MRT5005 was well tolerated at the 8-mg and 16-mg dose levels, and no serious side events were observed at any dose level. The lung function was evaluated by percent predicted forced expiratory volume in $1 \mathrm{~s}$ (PPFEV1). According to the interim report, the three patients who received the 16-mg dose showed maximal PPFEV1 increases of $11.1 \%, 13.6 \%$ and $22.2 \%$, respectively, on day 8 post nebulizing. mRNA-based protein replacement therapies are also being explored for heart ${ }^{241}$ liver $^{242}, \operatorname{lung}^{243}$ and other organ diseases ${ }^{12}$.

\section{Conclusions and future directions}

Progress in mRNA technologies and lipid nanoparticlebased delivery systems has allowed the development of mRNA COVID-19 vaccines at unprecedented speed, demonstrating the clinical potential of lipid nanoparticle-mRNA formulations and providing a powerful tool against the SARS-CoV-2 pandemic. A variety of lipid nanoparticles have been explored and optimized for mRNA delivery, providing valuable information for the future design of mRNA therapeutics. Based on the lessons and experiences from clinical studies, lipid nanoparticle-mRNA formulations can be further improved.

The in vivo translation efficiency of mRNA molecules could be further increased by RNA engineering. To achieve effective translation, mRNA requires five structural elements, including the $5^{\prime}$ cap, $3^{\prime}$ poly(A) tail, protein-coding sequence and $5^{\prime}$ and $3^{\prime}$ untranslated regions (UTRs) ${ }^{2,4}$. The sequences of these elements regulate translation initiation, translation termination and post-transcriptional modification of mRNA molecules ${ }^{2,4}$. Thus, sequence engineering of these elements could improve translation in vivo. For example, optimization of the UTRs or the coding sequences results in increased protein expression, compared with wild-type controls ${ }^{107,244}$. In addition, circular RNA (circRNA) can be synthesized to optimize mRNA properties ${ }^{245,246}$ (BOX 2). circRNA lacks the free ends necessary for nuclease-mediated degradation and, therefore, has a longer half-life than its linear mRNA counterpart ${ }^{245,246}$.

Moreover, the delivery efficacy of mRNA could be improved, for example, by rational design of lipids through modulation of head groups and hydrophobic tails to increase cellular uptake and endosomal escape of lipid nanoparticle-mRNA formulations $\mathrm{s}^{64,66,76-78,88,97,100,114,140,141}$. Furthermore, hybrid nanoparticles may integrate the advantages of individual components to improve mRNA delivery potency. For example, $\mathrm{pH}$-responsive polymers, such as poly ( $\beta$-amino ester), can be incorporated into lipid nanoparticles to facilitate endosomal escape of mRNA molecules ${ }^{160}$. Polymers, such as polyethyleneimine, protamine and polyaspartamide derivatives, are already widely used for mRNA delivery ${ }^{7-17}$. 
In addition, charge-altering releasable transporters ${ }^{162,247}$ and modified dendrimers ${ }^{248}$ can effectively deliver mRNA molecules in vitro and in vivo. Naturally derived membrane lipids (for example, exosomes and cell membranes) can also be applied for mRNA delivery ${ }^{116,249,250}$.

Organ-specific and cell-specific delivery of lipid nanoparticles can be achieved by modulating the lipid structures. For example, alteration of the alkyl length of a lipid results in selective accumulation of lipid nanoparticle-mRNA formulations in the liver or spleen ${ }^{114}$. Alternatively, biomimetic lipids can be designed to achieve organ-targeted delivery. For example, neurotransmitters are endogenous chemicals that can cross the blood-brain barrier and participate in neurotransmission ${ }^{82}$. Thus, neurotransmitter-derived lipids can be used for mRNA delivery to the brain following i.v. injection ${ }^{82}$. Testing and comparing the cell distribution of many different lipid nanoparticle formulations remains challenging. However, barcoded nanoparticles allow in vivo high-throughput profiling of lipid nanoparticle distribution at the cell level ${ }^{121}$. For example, barcoding has been applied to study how the structure of cholesterol derivatives impact cell selectivity of lipid nanoparticles, revealing that selective accumulation in liver endothelial cells, Kupffer cells and hepatocytes ${ }^{125,126}$ depends on cholesterol structures. Such large data sets will pave the way for a more profound understanding of the relationship between lipid nanoparticle properties and biodistribution.

Finally, biodegradability and multifunctionality should be considered for the design of lipid nanoparticles. Biodegradable lipids enable fast elimination of lipid nanoparticles from plasma and tissues, improving their safety and tolerability. Notably, biodegradable lipids are part of the mRNA-1273 and BNT162b2 COVID-19 mRNA vaccines. In addition to serving as delivery component, lipids may have therapeutic effects synergistic with mRNA-encoded proteins. Such multifunctional lipid materials include self-adjuvant lipids, which boost vaccine efficacy ${ }^{117,119}$, and paclitaxel-derived lipids, which allow integration of chemotherapies and gene therapies for the treatment of cancer ${ }^{118}$.

In summary, mRNA has shown great therapeutic potential in a number of clinical trials and in clinical applications. The development of next-generation lipid nanoparticles and other types of delivery material will further enable mRNA-based therapies for a broad range of diseases and improve health care in the near future.

Published online 10 August 2021
1. Cobb, M. Who discovered messenger RNA? Curr. Biol. 25, R526-R532 (2015).

2. Sahin, U., Karikó, K. \& Türeci, Ö. mRNA-based therapeutics - developing a new class of drugs Nat. Rev. Drug Discov. 13, 759-780 (2014). Wolff, J. A. et al. Direct gene transfer into mouse muscle in vivo. Science 247, 1465-1468 (1990)

4. Pardi, N., Hogan, M. J., Porter, F. W. \& Weissman, D. mRNA vaccines - a new era in vaccinology. Nat. Rev. Drug Discov. 17, 261-279 (2018).

5. Langer, R. \& Folkman, J. Polymers for the sustained release of proteins and other macromolecules. Nature 263, 797-800 (1976).

6. Langer, R. Controlling the movement of molecules. Q. Rev. Biophys. https://doi.org/10.1017/ S0033583519000040 (2019)

7. Hajj, K. A. \& Whitehead, K. A. Tools for translation: non-viral materials for therapeutic mRNA delivery. Nat. Rev. Mater. 2, 17056 (2017).

8. Xiong, Q., Lee, G. Y., Ding, J., Li, W. \& Shi, J. Biomedical applications of mRNA nanomedicine. Nano Res. 11, 5281-5309 (2018).

9. Guan, S. \& Rosenecker, J. Nanotechnologies in delivery of mRNA therapeutics using nonviral vector-based delivery systems. Gene Ther. $\mathbf{2 4}$ 133-143 (2017)

10. Li, B., Zhang, X. \& Dong, Y. Nanoscale platforms for messenger RNA delivery. Wiley Interdiscip. Rev. Nanomed. Nanobiotechnol. 11, e1530 (2019).

11. Kowalski, P. S., Rudra, A., Miao, L. \& Anderson, D. G. Delivering the messenger: advances in technologies for therapeutic mRNA delivery. Mol. Ther. 27, 710-728 (2019).

12. Zhao, W., Hou, X., Vick, O. G. \& Dong, Y. RNA delivery biomaterials for the treatment of genetic and rare diseases. Biomaterials 217 , 119291 (2019)

13. Uchida, S., Perche, F., Pichon, C. \& Cabral, H Nanomedicine-based approaches for mRNA delivery. Mol. Pharm. 17, 3654-3684 (2020).

14. Meng, C., Chen, Z., Li, G., Welte, T. \& Shen, H Nanoplatforms for mRNA therapeutics. Adv. Ther. 4 2000099 (2021)

15. Gebre, M. S. et al. Novel approaches for vaccine development. Cell 184, 1589-1603 (2021).

16. Weng, Y. et al. The challenge and prospect of mRNA therapeutics landscape. Biotechnol. Adv. 40, 107534 (2020).

17. Kim, J., Eygeris, Y., Gupta, M. \& Sahay, G. Self-assembled mRNA vaccines. Adv. Drug Deliv. Rev. 170, 83-112 (2021).

18. Akinc, A. et al. The Onpattro story and the clinical translation of nanomedicines containing nucleic acid-based drugs. Nat. Nanotechnol. 14, 1084-1087 (2019).

The first approved siRNA drug delivered by lipid nanoparticles.

19. Baden, L. R. et al. Efficacy and safety of the mRNA-1273 SARS-CoV-2 vaccine. N. Engl. J. Med. 384, 403-416 (2021).

20. Anderson, E. J. et al. Safety and immunogenicity of SARS-CoV-2 mRNA-1273 vaccine in older adults. N. Engl. J. Med. 383, 2427-2438 (2020).

21. Polack, F. P. et al. Safety and efficacy of the BNT162b2 mRNA Covid-19 vaccine. N. Engl. J. Med. 383, 2603-2615 (2020).

References 19, 20, and 21 report safety and efficacy data about the SARS-CoV-2 lipid nanoparticlemRNA vaccines BNT162b2 and mRNA-1273.

22. Ostro, M. J., Giacomoni, D., Lavelle, D., Paxton, W. $\&$ Dray, S. Evidence for translation of rabbit globin mRNA after liposomemediated insertion into a human cell line. Nature 274, 921-923 (1978).

23. Dimitriadis, G. J. Translation of rabbit globin mRNA introduced by liposomes into mouse lymphocytes. Nature 274, 923-924 (1978)

24. Malone, R. W., Felgner, P. L. \& Verma, I. M. Cationic liposome-mediated RNA transfection. Proc. Natl Acad. Sci. USA 86, 6077-6081 (1989).

25. Felgner, J., Martin, M., Tsai, Y. \& Felgner, P. L. Cationic lipid-mediated transfection in mammalian cells: "Lipofection". J. Tissue Cult. Methods 15, 63-68 (1993).

26. Kranz, L. M. et al. Systemic RNA delivery to dendritic cells exploits antiviral defence for cancer immunotherapy. Nature 534, 396-401 (2016).

27. Krienke, $\mathrm{C}$. et al. A noninflammatory mRNA vaccine for treatment of experimental autoimmune encephalomyelitis. Science 371, 145-153 (2021).

28. Brito, L. A. et al. A cationic nanoemulsion for the delivery of next-generation RNA vaccines. Mol. Ther 22, 2118-2129 (2014)

29. Brazzoli, M. et al. Induction of broad-based immunity and protective efficacy by self-amplifying mRNA vaccines encoding influenza virus hemagglutinin. J. Virol. 90, 332-344 (2016).

30. Maruggi, G. et al. Immunogenicity and protective efficacy induced by self-amplifying mRNA vaccines encoding bacterial antigens. Vaccine 35, 361-368 (2017).

31. Garcia, A. B. et al. Neutralization of the Plasmodiumencoded MIF ortholog confers protective immunity against malaria infection. Nat. Commun. 9, 2714 (2018).

32. Yang, J. et al. Hybrid nanovaccine for the co-delivery of the mRNA antigen and adjuvant. Nanoscale 11, 21782-21789 (2019).
33. Lei, S. et al. Efficient colorectal cancer gene therapy with IL-15 mRNA nanoformulation. Mol. Pharm. 17. 3378-3391 (2020).

34. Mai, Y. et al. Intranasal delivery of cationic liposomeprotamine complex mRNA vaccine elicits effective anti-tumor immunity. Cell Immunol. 354, 104143 (2020).

35. Wang, Y. et al. Systemic delivery of modified mRNA encoding herpes simplex virus 1 thymidine kinase for targeted cancer gene therapy. Mol. Ther. 21, 358-367 (2013).

36. Zhang, R. et al. Delivery of a modified mRNA encoding IL-22 binding protein (IL-22BP) for colon cancer gene therapy. J. Biomed. Nanotechnol. 14, 1239-1251 (2018).

37. Lara, H. et al. Targeting serous epithelial ovarian cancer with designer zinc finger transcription factors. J. Biol. Chem. 287, 29873-29886 (2012).

38. Erasmus, J. H. et al. A nanostructured lipid carrier for delivery of a replicating viral RNA provides single, low-dose protection against Zika. Mol. Ther. 26, 2507-2522 (2018).

39. Erasmus, J. H. et al. Intramuscular delivery of replicon RNA encoding ZIKV-117 human monoclonal antibody protects against Zika virus infection. Mol. Ther. Methods Clin. Dev. 18, 402-414 (2020).

40. Erasmus, J. H. et al. An Alphavirus-derived replicon RNA vaccine induces SARS-CoV- 2 neutralizing antibody and $\mathrm{T}$ cell responses in mice and nonhuman primates. Sci. Transl Med. 12, eabc9396 (2020).

41. Perche, F. et al. Neutral lipopolyplexes for in vivo delivery of conventional and replicative RNA vaccine. Mol. Ther. Nucleic Acids 17, 767-775 (2019).

42. Prieve, M. G. et al. Targeted mRNA therapy for ornithine transcarbamylase deficiency. Mol. Ther. 26, 801-813 (2018)

43. Zohra, F. T., Chowdhury, E. H. \& Akaike, T. High performance mRNA transfection through carbonate apatite-cationic liposome conjugates. Biomaterials 30, 4006-4013 (2009).

44. Zohra, F. T., Maitani, Y. $\&$ Akaike, T. mRNA delivery through fibronectin associated liposome-apatite particles: a new approach for enhanced mRNA transfection to mammalian cell. Biol. Pharm. Bull. 35 111-115 (2012).

45. Hilgers, L. $\&$ Snippe, H. DDA as an immunological adjuvant. Res. Immunol. 143, 494-503 (1992). Discussion 574-6.

46. Blakney, A. K., McKay, P. F., Yus, B. I., Aldon, Y. $\&$ Shattock, R. J. Inside out: optimization of lipid nanoparticle formulations for exterior complexation and in vivo delivery of saRNA. Gene Ther. 26, 363-372 (2019). 
47. Lou, G. et al. Delivery of self-amplifying mRNA vaccines by cationic lipid nanoparticles: The impact of cationic lipid selection. J. Control. Release 325 , 370-379 (2020)

48. Kormann, M. S. et al. Expression of therapeutic proteins after delivery of chemically modified mRNA in mice Nat Biotechnol 29,154-157 (2011).

49. Zangi, L. et al. Modified mRNA directs the fate of heart progenitor cells and induces vascular regeneration after myocardial infarction. Nat. Biotechnol. 31, 898-907 (2013).

50. Warren, L. et al. Highly efficient reprogramming to pluripotency and directed differentiation of human cells with synthetic modified mRNA. Cell Stem Cell 7 618-630 (2010).

51. Fang, N., Wang, J., Mao, H.-O., Leong, K. W. \& Chan, V. BHEM-Chol/DOPE liposome induced perturbation of phospholipid bilayer. Colloid Surf. B Biointerfaces 29 233-245 (2003)

52. Fan, Y.-N. et al. Cationic lipid-assisted nanoparticles for delivery of mRNA cancer vaccine. Biomater. Sci. 6, 3009-3018 (2018)

53. Zhang, Y. et al. In situ repurposing of dendritic cells with CRISPR/Cas9-based nanomedicine to induce transplant tolerance. Biomaterials 217, 119302 (2019).

54. Persano, S. et al. Lipopolyplex potentiates anti-tumor immunity of mRNA-based vaccination. Biomaterials 125, 81-89 (2017)

55. Zhang, J., Shrivastava, S., Cleveland, R. O. \& Rabbitts, T. H. Lipid-mRNA nanoparticle designed to enhance intracellular delivery mediated by shock waves ACS Appl. Mater. Interfaces 11, 10481-10491 (2019).

56. Patel, S., Ryals, R. C., Weller, K. K., Pennesi, M. E. \& Sahay, G. Lipid nanoparticles for delivery of messenge RNA to the back of the eye. J. Control. Release 303 , 91-100 (2019)

57. Behr, J.-P., Demeneix, B., Loeffler, J.-P. \& Perez-Mutul, J. Efficient gene transfer into mammalian primary endocrine cells with lipopolyamine-coated DNA Proc. Natl Acad. Sci. USA 86, 6982-6986 (1989).

58. Ewert, K., Ahmad, A., Evans, H. M., Schmidt, H.-W. \& Safinya, C. R. Efficient synthesis and cell-transfection properties of a new multivalent cationic lipid for nonviral gene delivery. J. Med. Chem. 45, 5023-5029 (2002).

59. Gao, X. \& Huang, L. A novel cationic liposome reagent for efficient transfection of mammalian cells. Biochem. Biophys. Res. Commun. 179, 280-285 (1991).

60. Lee, E. R. et al. Detailed analysis of structures and formulations of cationic lipids for efficient gene transfer to the lung. Hum. Gene Ther. 7, 1701-1717 (1996).

61. Zhou, W.-Z. et al. RNA melanoma vaccine: induction of antitumor immunity by human glycoprotein 100 mRNA immunization. Hum. Gene Ther. 10, 2719-2724 (1999).

62. Andries, O. et al. Innate immune response and programmed cell death following carrier-mediated delivery of unmodified mRNA to respiratory cells. J. Control. Release 167, 157-166 (2013).

63. Guevara, M. L., Jilesen, Z., Stojdl, D. \& Persano, S Codelivery of mRNA with a-galactosylceramide using a new lipopolyplex formulation induces a strong antitumor response upon intravenous administration. ACS Omega 4, 13015-13026 (2019).

64. Heyes, J., Palmer, L., Bremner, K. \& MacLachlan, I. Cationic lipid saturation influences intracellular delivery of encapsulated nucleic acids. J. Control. Release 107, 276-287 (2005)

65. Semple, S. C. et al. Rational design of cationic lipids for siRNA delivery. Nat. Biotechnol. 28, 172-176 (2010).

66. Jayaraman, M. et al. Maximizing the potency of siRNA lipid nanoparticles for hepatic gene silencing in vivo. Angew. Chem. 124, 8657-8661 (2012).

67. Nabhan, J. F. et al. Intrathecal delivery of frataxin mRNA encapsulated in lipid nanoparticles to dorsal root ganglia as a potential therapeutic for Friedreich's ataxia. Sci. Rep. 6, 20019 (2016)

68. Robinson, E. et al. Lipid nanoparticle-delivered chemically modified mRNA restores chloride secretion in cystic fibrosis. Mol. Ther. 26, 2034-2046 (2018).

69. Veiga, N. et al. Cell specific delivery of modified mRNA expressing therapeutic proteins to leukocytes. Nat. Commun. 9, 4493 (2018)

70. Jain, R. et al. MicroRNAs enable mRNA therapeutics to selectively program cancer cells to self-destruct. Nucleic Acid Ther. 28, 285-296 (2018)

71. Zhang, H., Leal, J., Soto, M. R., Smyth, H. D. \& Ghosh, D Aerosolizable lipid nanoparticles for pulmonary delivery of mRNA through design of experiments. Pharmaceutics 12, 1042 (2020).

72. Ryals, R. C. et al. The effects of PEGylation on LNP based mRNA delivery to the eye. PLOS ONE 15 e0241006 (2020).
73. Zhang, M., Sun, J., Li, M. \& Jin, X. Modified mRNALNP vaccines confer protection against experimental DENV-2 infection in mice. Mol. Ther. Methods Clin. Dev. 18, 702-712 (2020)

74. Lu, J. et al. A COVID-19 mRNA vaccine encoding SARS-CoV-2 virus-like particles induces a strong antiviral-like immune response in mice. Cell Res. 30 936-939 (2020).

75. Kim, J., Mukherjee, A., Nelson, D., Jozi c, A. \& Sahay, C. Rapid generation of circulating and mucosal decoy ACE2 using mRNA nanotherapeutics for the potential treatment of SARS-CoV-2 Preprint at bioRxiv https:// doi.org/10.1101/2020.07.24.205583 (2020).

76. Maier, M. A. et al. Biodegradable lipids enabling rapidly eliminated lipid nanoparticles for systemic delivery of RNAi therapeutics. Mol. Ther. $\mathbf{2 1}$ 1570-1578 (2013).

77. Sabnis, S. et al. A novel amino lipid series for mRNA delivery: improved endosomal escape and sustained pharmacology and safety in non-human primates. Mol. Ther. 26, 1509-1519 (2018)

78. Hassett, K. J. et al. Optimization of lipid nanoparticles for intramuscular administration of mRNA vaccines. Mol. Ther. Nucleic Acids 15, 1-11 (2019).

79. Ansell, S. M. \& Du, X. Lipids and lipid nanoparticle formulations for delivery of nucleic acids. US Patent US10166298B2 (2019).

80. Tanaka, H. et al. Self-degradable lipid-like materials based on "hydrolysis accelerated by the intra-particle enrichment of reactant (HyPER)" for messenger RNA delivery. Adv. Funct. Mater. 30, 1910575 (2020).

81. Liu, J. et al. Fast and efficient CRISPR/Cas9 genome editing in vivo enabled by bioreducible lipid and messenger RNA nanoparticles. Adv. Mater. 31 1902575 (2019)

82. Ma, F. et al. Neurotransmitter-derived lipidoids (NT-lipidoids) for enhanced brain delivery through intravenous injection. Sci. Adv. 6, eabb4429 (2020).

83. Zhao, X. et al. mRNA delivery using bioreducible lipidoid nanoparticles facilitates neural differentiation of human mesenchymal stem cells. Adv. Healthc. Mater. 10, 2000938 (2021)

84. Zhao, X. et al. Imidazole-based synthetic lipidoids for in vivo mRNA delivery into primary T lymphocytes. Angew. Chem. 132, 20258-20264 (2020).

85. Qiu, M. et al. Lipid nanoparticle-mediated codelivery of Cas9 mRNA and single-guide RNA achieves liverspecific in vivo genome editing of Angptl3. Proc. Natl Acad. Sci. USA 118, e2020401118 (2021).

86. Akinc, A. et al. A combinatorial library of lipid-like materials for delivery of RNAi therapeutics. Nat. Biotechnol. 26, 561-569 (2008).

87. Love, K. T. et al. Lipid-like materials for low-dose, in vivo gene silencing. Proc. Natl Acad. Sci. USA 107, 1864-1869 (2010).

88. Whitehead, K. A. et al. Degradable lipid nanoparticles with predictable in vivo siRNA delivery activity. Nat. Commun. 5, 4277 (2014).

89. Dong, Y. et al. Lipopeptide nanoparticles for potent and selective siRNA delivery in rodents and nonhuman primates. Proc. Natl Acad. Sci. USA 111, 3955-3960 (2014).

90. Kauffman, K. J. et al. Optimization of lipid nanoparticle formulations for mRNA delivery in vivo with fractional factorial and definitive screening designs. Nano Lett. 15, 7300-7306 (2015).

91. DeRosa, F. et al. Therapeutic efficacy in a hemophilia model using a biosynthetic mRNA liver depot system. Gene Ther. 23, 699-707 (2016)

92. Yin, H. et al. Therapeutic genome editing by combined viral and non-viral delivery of CRISPR system components in vivo. Nat. Biotechnol. 34, 328-333 (2016).

93. Turnbull, I. C. et al. Myocardial delivery of lipidoid nanoparticle carrying modRNA induces rapid and transient expression. Mol. Ther. 24, 66-75 (2016)

94. Oberli, M. A. et al. Lipid nanoparticle assisted mRNA delivery for potent cancer immunotherapy. Nano Lett. 17, 1326-1335 (2017)

95. Rybakova, Y. et al. mRNA delivery for therapeutic anti-HER2 antibody expression in vivo. Mol. Ther. $\mathbf{2 7}$ 1415-1423 (2019).

96. Yin $\mathrm{H}$ et al Structure-guided chemical modification of guide RNA enables potent non-viral in vivo genome editing. Nat. Biotechnol. 35, 1179-1187 (2017)

97. Hajj, K. A. et al. Branched-tail lipid nanoparticles potently deliver mRNA in vivo due to enhanced ionization at endosomal pH. Small 15, 1805097 (2019).

98. Hajj, K. A. et al. A potent branched-tail lipid nanoparticle enables multiplexed mRNA delivery and gene editing in vivo. Nano Lett. 20,5167-5175 (2020).
99. Ball, R. L., Haij, K. A., Vizelman, J., Bajaj, P. \& Whitehead, K. A. Lipid nanoparticle formulations for enhanced co-delivery of siRNA and mRNA. Nano Lett. 18, 3814-3822 (2018)

100. Miao, L. et al. Synergistic lipid compositions for albumin receptor mediated delivery of mRNA to the liver. Nat. Commun. 11, 2424 (2020).

101. Fenton, O. S. et al. Bioinspired alkenyl amino alcohol ionizable lipid materials for highly potent in vivo mRNA delivery. Adv. Mater. 28, 2939-2943 (2016).

102. Fenton, O. S. et al. Synthesis and biological evaluation of ionizable lipid materials for the in vivo delivery of messenger RNA to B lymphocytes. Adv. Mater. 29, 1606944 (2017)

103. Fenton, O. S. et al. Customizable lipid nanoparticle materials for the delivery of siRNAs and mRNAs. Angew. Chem. Int Ed. 57, 13582-13586 (2018).

104. Li, B. et al. An orthogonal array optimization of lipid-like nanoparticles for mRNA delivery in vivo. Nano Lett. 15, 8099-8107 (2015).

105. Jiang, C. et al. A non-viral CRISPR/Cas9 delivery system for therapeutically targeting HBV DNA and pcsk9 in vivo. Cell Res. 27, 440-443 (2017).

106. Li, Y. et al. Multifunctional oncolytic nanoparticles deliver self-replicating IL-12 RNA to eliminate established tumors and prime systemic immunity. Nat. Cancer 1, 882-893 (2020).

107. Zeng, C. et al. Leveraging mRNA sequences and nanoparticles to deliver SARS-CoV-2 antigens in vivo. Adv. Mater. 32, 2004452 (2020).

108. Zhang, X. et al. Functionalized lipid-like nanoparticles for in vivo mRNA delivery and base editing. Sci. Adv. 6, eabc2315 (2020).

109. Colombani, T. et al. Self-assembling complexes between binary mixtures of lipids with different linkers and nucleic acids promote universal mRNA, DNA and siRNA delivery. J. Control. Release 249, 131-142 (2017).

110. Habrant, D. et al. Design of ionizable lipids to overcome the limiting step of endosomal escape: application in the intracellular delivery of mRNA DNA and siRNA. J. Med. Chem. 59, 3046-3062 (2016).

111. Yu, X. et al. Lipid-modified aminoglycosides for mRNA delivery to the liver. Adv. Healthc. Mater. 9, 1901487 (2020).

112. Zhang, Y. et al. Lipid-modified aminoglycoside derivatives for in vivo siRNA delivery. Adv. Mater. 25 4641-4645 (2013).

113. Miller, J. B. et al. Non-viral CRISPR/Cas gene editing in vitro and in vivo enabled by synthetic nanoparticle co-delivery of Cas9 mRNA and sgRNA. Angew. Chem. Int. Ed. 56, 1059-1063 (2017).

114. Liu, S. et al. Membrane-destabilizing ionizable phospholipids for organ-selective mRNA delivery and CRISPR-Cas gene editing. Nat. Mater. 20, 701-710 (2021).

115. Walsh, C. L., Nguyen, J. \& Szoka, F. C. Synthesis and characterization of novel zwitterionic lipids with pH-responsive biophysical properties. Chem. Commun. 48, 5575-5577 (2012).

116. Kim, J., Jozic, A. \& Sahay, G. Naturally derived membrane lipids impact nanoparticle-based messenger RNA delivery. Cell. Mol. Bioeng. 13, 463-474 (2020)

117. Miao, L. et al. Delivery of mRNA vaccines with heterocyclic lipids increases anti-tumor efficacy by STING-mediated immune cell activation. Nat. Biotechnol. 37, 1174-1185 (2019).

118. Zhang, C. et al. Chemotherapy drugs derived nanoparticles encapsulating mRNA encoding tumor suppressor proteins to treat triple-negative breast cancer. Nano Res. 12, 855-861 (2019).

119. Zhang, H. et al. Delivery of mRNA vaccine with a lipidlike material potentiates antitumor efficacy through Toll-like receptor 4 signaling. Proc. Natl Acad. Sci. USA 118, e2005191118 (2021).

120. Koltover I Salditt, T., Rädler, J O \& Safinya, C. R. An inverted hexagonal phase of cationic liposomeDNA complexes related to DNA release and delivery. Science 281, 78-81 (1998)

121. Dahlman, J. E. et al. Barcoded nanoparticles for high throughput in vivo discovery of targeted therapeutics. Proc. Natl Acad. Sci. USA 114, 2060-2065 (2017).

122. Gan, Z. et al. Nanoparticles containing constrained phospholipids deliver mRNA to liver immune cells in vivo without targeting ligands. Bioeng. Transl Med. 5, e10161 (2020)

123. Patel, S. et al. Naturally-occurring cholestero analogues in lipid nanoparticles induce polymorphic shape and enhance intracellular delivery of mRNA. Nat Commun. 11, 983 (2020). 
124. Eygeris, Y., Patel, S., Jozic, A. \& Sahay, C. Deconvoluting lipid nanoparticle structure for messenger RNA delivery. Nano Lett. 20, 4543-4549 (2020).

125. Paunovska, K. et al. Analyzing 2000 in vivo drug delivery data points reveals cholesterol structure impacts nanoparticle delivery. ACS Nano 12 8341-8349 (2018).

126. Paunovska, K. et al. Nanoparticles containing oxidized cholesterol deliver mRNA to the live microenvironment at clinically relevant doses. Adv. Mater. 31, 1807748 (2019).

127. Knop, K., Hoogenboom, R., Fischer, D. \& Schubert, U. S Poly(ethylene glycol) in drug delivery: pros and cons as well as potential alternatives. Angew. Chem. Int. Ed. 49, 6288-6308 (2010).

128. Akinc, A. et al. Development of lipidoid-siRNA formulations for systemic delivery to the liver. Mol. Ther. 17, 872-879 (2009).

129. Zhu, X. et al. Surface De-PEGylation controls nanoparticle-mediated siRNA delivery in vitro and in vivo. Theranostics $7,1990-2002$ (2017).

130. Karnik, R. et al. Microfluidic platform for controlled synthesis of polymeric nanoparticles. Nano Lett. 8, 2906-2912 (2008)

131. Leung, A. K., Tam, Y. Y. C., Chen, S., Hafez, I. M. \& Cullis, P. R. Microfluidic mixing: a general method for encapsulating macromolecules in lipid nanoparticle systems. J. Phys. Chem. B 119, 8698-8706 (2015)

132. Kedmi, R. et al. A modular platform for targeted RNA therapeutics. Nat. Nanotechnol. 13, 214-219 (2018)

133. Rosenblum, D. et al. CRISPR-Cas9 genome editing using targeted lipid nanoparticles for cancer therapy. Sci. Adv. 6, eabc9450 (2020).

134. Cheng, Q. et al. Selective organ targeting (SORT) nanoparticles for tissue-specific mRNA delivery and CRISPR-Cas gene editing. Nat. Nanotechnol. 15, 313-320 (2020)

135. Kim, M. et al. Engineered ionizable lipid nanoparticles for targeted delivery of RNA therapeutics into differen types of cells in the liver. Sci. Adv. 7, eabf4398 (2021).

136. Hou, X. et al. Vitamin lipid nanoparticles enable adoptive macrophage transfer for the treatment of multidrug-resistant bacterial sepsis. Nat. Nanotechnol. 15, 41-46 (2020).

137. Sahay, G. et al. Efficiency of siRNA delivery by lipid nanoparticles is limited by endocytic recycling. Nat. Biotechnol. 31, 653-658 (2013).

138. Gilleron, J. et al. Image-based analysis of lipid nanoparticle-mediated siRNA delivery, intracellular trafficking and endosomal escape. Nat. Biotechnol. 31, 638-646 (2013)

139. Wittrup, A. et al. Visualizing lipid-formulated siRNA release from endosomes and target gene knockdown. Nat. Biotechnol. 33, 870-876 (2015).

140. Alabi, C. A. et al. Multiparametric approach for the evaluation of lipid nanoparticles for siRNA delivery. Proc. Natl Acad. Sci. USA 110, 12881-12886 (2013).

141. Lee, S. M. et al. A systematic study of unsaturation in lipid nanoparticles leads to improved mRNA transfection in vivo. Angew. Chem. Int. Ed. 60 5848-5853 (2021).

142. Cheng, Q. et al. Dendrimer-based lipid nanoparticles deliver therapeutic FAH mRNA to normalize liver function and extend survival in a mouse model of hepatorenal tyrosinemia type I. Adv. Mater. 30 1805308 (2018)

143. Pardi, N. et al. Expression kinetics of nucleosidemodified mRNA delivered in lipid nanoparticles to mice by various routes. J. Control. Release 217, 345-351 (2015)

144. Melo, M. et al. Immunogenicity of RNA replicons encoding HIV Env immunogens designed for self-assembly into nanoparticles. Mol. Ther. 27, 2080-2090 (2019).

145. Pardi, N. et al. Administration of nucleoside-modified mRNA encoding broadly neutralizing antibody protects humanized mice from HIV-1 challenge. Nat. Commun. 8, 14630 (2017).

146. Thran, M. et al. mRNA mediates passive vaccination against infectious agents, toxins, and tumors. EMBO Mol. Med. 9, 1434-1447 (2017).

147. Stadler, C. R. et al. Elimination of large tumors in mice by mRNA-encoded bispecific antibodies. Nat. Med. 23, 815-817 (2017).

148. Sayour, E. J. et al. Systemic activation of antigenpresenting cells via RNA-loaded nanoparticles. Oncoimmunology 6, e1256527 (2017).

149. Broos, K. et al. Particle-mediated intravenous delivery of antigen mRNA results in strong antigen-specific
T-cell responses despite the induction of type I interferon. Mol. Ther. Nucleic Acids 5, e326 (2016).

150. Van der Jeught, K. et al. Dendritic cell targeting mRNA lipopolyplexes combine strong antitumor T-cell immunity with improved inflammatory safety. ACS Nano 12, 9815-9829 (2018).

151. Devoldere, J. et al. Non-viral delivery of chemically modified mRNA to the retina: Subretinal versus intravitreal administration. J. Control. Release 307 315-330 (2019).

152. Anderson, D. M. et al. Stability of mRNA/cationic lipid lipoplexes in human and rat cerebrospinal fluid: methods and evidence for nonviral mRNA gene delivery to the central nervous system. Hum. Gene Ther. 14, 191-202 (2003).

153. Gan, L.-M. et al. Intradermal delivery of modified mRNA encoding VEGF-A in patients with type 2 diabetes. Nat. Commun. 10, 871 (2019).

154. Anttila, V. et al. Synthetic mRNA encoding VEGF-A in patients undergoing coronary artery bypass grafting: design of a phase 2 a clinical trial. Mol. Ther. Methods Clin. Dev. 18, 464-472 (2020)

155. Zeng, C., Zhang, C., Walker, P. G. \& Dong, Y. Formulation and delivery technologies for mRNA vaccines. Curr. Top. Microbiol. Immunol. https:// doi.org/10.1007/82_2020_217 (2020).

156. Bahl, K. et al. Preclinical and clinical demonstration of immunogenicity by mRNA vaccines against H10N8 and H7N9 influenza viruses. Mol. Ther. 25 1316-1327 (2017).

157. Feldman, R. A. et al. mRNA vaccines against $\mathrm{H} 10 \mathrm{~N} 8$ and H7N9 influenza viruses of pandemic potential are immunogenic and well tolerated in healthy adults in phase 1 randomized clinical trials. Vaccine 37 , 3326-3334 (2019).

This paper reports a clinical trial on lipid nanoparticle-mRNA formulations as influenza vaccines.

158. Phua, K. K., Staats, H. F., Leong, K. W. \& Nair, S. K. Intranasal mRNA nanoparticle vaccination induces prophylactic and therapeutic anti-tumor immunity. Sci. Rep. 4, 5128 (2014).

159. Zhuang, X. et al. mRNA vaccines encoding the HA protein of influenza A H1N1 virus delivered by cationic lipid nanoparticles induce protective immune responses in mice. Vaccines 8, 123 (2020).

160. Su, X., Fricke, J., Kavanagh, D. G. \& Irvine, D. J. In vitro and in vivo mRNA delivery using lipidenveloped $\mathrm{pH}$-responsive polymer nanoparticles. Mol. Pharm. 8, 774-787 (2011)

161. Li, Y. et al. In vitro evolution of enhanced RNA replicons for immunotherapy. Sci. Rep. 9, 6932 (2019).

162. Haabeth, O. A. W. et al. Local delivery of Ox40I, Cd80, and Cd86 mRNA kindles global anticance immunity. Cancer Res. 79, 1624-1634 (2019).

163. Hewitt, S. L. et al. Durable anticancer immunity from intratumoral administration of IL-23, IL-36y, and OX40L mRNAs. Sci. Transl Med. 11, eaat9143 (2019).

164. Riley, R. S. et al. Ionizable lipid nanoparticles for in utero mRNA delivery. Sci. Adv. 7, eaba 1028 (2021).

165. Von Der Mülbe, F. et al. Method for producing RNA US Patent US10017826B2 (2018).

166. DeRosa, F. et al. Methods for purification of messenger RNA. US Patent US10975369B2 (2021).

167. MacLachlan, I. Liposomal formulations for nucleic acid delivery. Antis. Drug Technol. 2, 237-270 (2007)

168. Zhao, P. et al. Long-term storage of lipid-like nanoparticles for mRNA delivery. Bioact. Mater. $\mathbf{5}$, 358-363 (2020)

169. Lila, A. S. A., Kiwada, H. \& Ishida, T. The accelerated blood clearance $(A B C)$ phenomenon: clinical challenge and approaches to manage. J. Control. Release 172, 38-47 (2013)

170. Kedmi, R., Ben-Arie, N. \& Peer, D. The systemic toxicity of positively charged lipid nanoparticles and the role of Toll-like receptor 4 in immune activation. Biomaterials 31, 6867-6875 (2010)

171. Dokka, S., Toledo, D., Shi, X., Castranova, V. \& Rojanasakul, Y. Oxygen radical-mediated pulmonary toxicity induced by some cationic liposomes. Pharm. Res. 17, 521-525 (2000)

172. Abrams, M. T. et al. Evaluation of efficacy, biodistribution, and inflammation for a potent siRNA nanoparticle: effect of dexamethasone co-treatment. Mol. Ther. 18, 171-180 (2010).

173. Sedic, M. et al. Safety evaluation of lipid nanoparticleformulated modified mRNA in the Sprague-Dawley rat and cynomolgus monkey. Vet. Pathol. 55, 341-354 (2018).
174. Szebeni, J. Complement activation-related pseudoallergy: a stress reaction in blood triggered by nanomedicines and biologicals. Mol. Immunol. $\mathbf{6 1}$ 163-173 (2014)

175. Lokugamage, M. P. et al. Mild innate immune activation overrides efficient nanoparticle-mediated RNA delivery. Adv. Mater. 32, 1904905 (2020).

176. Lv, H., Zhang, S., Wang, B., Cui, S. \& Yan, J. Toxicity of cationic lipids and cationic polymers in gene delivery. J. Control. Release 114, 100-109 (2006).

177. Filion, M. C. \& Phillips, N. C. Toxicity and immunomodulatory activity of liposomal vectors formulated with cationic lipids toward immune effector cells. Biochim. Biophys. Acta 1329, 345-356 (1997).

178. Li, S. et al. Effect of immune response on gene transfer to the lung via systemic administration of cationic lipidic vectors. Am. J. Physiol. Lung Cell. Mol. Physiol. 276, L796-L804 (1999).

179. Zhang, X. et al. Biodegradable amino-ester nanomaterials for Cas9 mRNA delivery in vitro and in vivo. ACS Appl. Mater. Interfaces 9, 25481-25487 (2017).

180. Karikó, K. et al. Incorporation of pseudouridine into mRNA yields superior nonimmunogenic vector with increased translational capacity and biological stability. Mol. Ther. 16, 1833-1840 (2008).

181. Pollard, C et al Type I IFN counteracts the induction of antigen-specific immune responses by lipid-based delivery of mRNA vaccines. Mol. Ther. 21, 251-259 (2013).

182. Karikō, K., Buckstein, M., Ni, H. \& Weissman, D. Suppression of RNA recognition by Toll-like receptors: the impact of nucleoside modification and the evolutionary origin of RNA. Immunity 23, 165-175 (2005). This study demonstrates that nucleoside modifications reduce the immunogenecity of in vitro-transcribed $\mathrm{mRNA}$

183. Kariko, K., Muramatsu, H., Ludwig, J. \& Weissman, D. Generating the optimal mRNA for therapy: HPLC purification eliminates immune activation and improves translation of nucleoside-modified, proteinencoding mRNA. Nucleic Acids Res. 39, e142 (2011).

184. Martinon, F. et al. Induction of virus-specific cytotoxic T lymphocytes in vivo by liposome-entrapped mRNA. Eur. J. Immunol. 23, 1719-1722 (1993).

185. John, S. et al. Multi-antigenic human cytomegalovirus mRNA vaccines that elicit potent humoral and cellmediated immunity. Vaccine 36, 1689-1699 (2018)

186. McKay, P. F. et al. Self-amplifying RNA SARS-CoV-2 lipid nanoparticle vaccine candidate induces high neutralizing antibody titers in mice. Nat. Commun. 11 3523 (2020).

187. de Alwis, R. M. et al. A single dose of self-transcribing and replicating RNA-based SARS-CoV-2 vaccine produces protective adaptive immunity in mice. Mol. Ther. 29, 1970-1983 (2021)

188. Elia, U. et al. Design of SARS-CoV-2 hFc-conjugated receptor-binding domain $\mathrm{mRNA}$ vaccine delivered via lipid nanoparticles. ACS Nano 15, 9673-9637 (2021).

189. Pardi, N. et al. Nucleoside-modified mRNA immunization elicits influenza virus hemagglutinin stalkspecific antibodies. Nat. Commun. 9, 3361 (2018).

190. Pardi, N. et al. Nucleoside-modified mRNA vaccines induce potent $\mathrm{T}$ follicular helper and germinal center $\mathrm{B}$ cell responses. J. Exp. Med. 215, 1571-1588 (2018)

191. Goswami, R. et al. Mannosylation of LNP results in improved potency for self-amplifying RNA (SAM) vaccines. ACS Infect Dis. 5, 1546-1558 (2019)

192. Pardi, N. et al. Zika virus protection by a single lowdose nucleoside-modified mRNA vaccination. Nature 543, 248-251 (2017)

193. Richner J. M. et al. Vaccine mediated protection against Zika virus-induced congenital disease. Cell 170, 273-283.e12 (2017).

194. Richner, J. M. et al. Modified mRNA vaccines protect against Zika virus infection. Cell 168, 1114-1125.e10 (2017).

195. VanBlargan, L. A. et al. An mRNA vaccine protects mice against multiple tick-transmitted flavivirus infections. Cell Rep. 25, 3382-3392.e3 (2018).

196. Samsa, M. M. et al. Self-amplifying RNA vaccines for Venezuelan equine encephalitis virus induce robust protective immunogenicity in mice. Mol. Ther 27 850-865 (2019).

197. Meyer, M. et al. Modified mRNA-based vaccines elicit robust immune responses and protect guinea pigs from Ebola virus disease. J. Infect. Dis. 217, 451-455 (2018).

198. Arya, S., Lin, Q., Zhou, N., Gao, X. \& Huang, J.-D. Strong immune responses induced by direct local injections of modified mRNA-lipid nanocomplexes. Mol. Ther. Nucleic Acids 19, 1098-1109 (2020). 
199. Conry, R. M. et al. Characterization of a messenger RNA polynucleotide vaccine vector. Cancer Res. 55 , 1397-1400 (1995)

200. Sahin, U. et al. An RNA vaccine drives immunity in checkpoint-inhibitor-treated melanoma. Nature 585, 107-112 (2020).

This is a clinical trial on lipid nanoparticle-mRNA formulations for cancer immunotherapy.

201. Liu, L. et al. Combination immunotherapy of MUC1 mRNA nano-vaccine and CTLA-4 blockade effectively inhibits growth of triple negative breast cancer. Mol. Ther. 26, 45-55 (2018)

202. Wang, Y., Zhang, L., Xu, Z., Miao, L. \& Huang, L. mRNA vaccine with antigen-specific checkpoint blockade induces an enhanced immune response against established melanoma. Mol. Ther. 26, 420-434 (2018)

203. Verbeke, R. et al. Broadening the message: a nanovaccine co-loaded with messenger RNA and a-GalCer induces antitumor immunity through conventional and natural killer T cells. ACS Nano 13, 1655-1669 (2019).

204. Verbeke, R. et al. Co-delivery of nucleoside-modified mRNA and TLR agonists for cancer immunotherapy: Restoring the immunogenicity of immunosilent mRNA J. Control. Release 266, 287-300 (2017).

205. Islam, M. A. et al. Adjuvant-pulsed mRNA vaccine nanoparticle for immunoprophylactic and therapeutic tumor suppression in mice. Biomaterials 266 120431 (2021).

206. Tse, S.-W. et al. mRNA-encoded, constitutively active STINGV155M is a potent genetic adjuvant of antigen-specific $\mathrm{CD} 8{ }^{+} \mathrm{T}$ cell response. $\mathrm{Mol}$. Ther. 29 2227-2238 (2021)

207. Dewitte, H. et al. The potential of antigen and TriMix sonoporation using mRNA-loaded microbubbles for ultrasound-triggered cancer immunotherapy. J. Control. Release 194, 28-36 (2014).

208. Hess, P. R., Boczkowski, D., Nair, S. K., Snyder, D. \& Gilboa, E. Vaccination with mRNAs encoding tumorassociated antigens and granulocyte-macrophage colony-stimulating factor efficiently primes CTL responses, but is insufficient to overcome tolerance to a model tumor/self antigen. Cancer Immunol. Immunother. 55, 672-683 (2006).

209. Sahin, U. et al. Personalized RNA mutanome vaccines mobilize poly-specific therapeutic immunity against cancer. Nature 547, 222-226 (2017)

210. Bauman, J. et al. 798 Safety, tolerability, and immunogenicity of mRNA-4157 in combination with pembrolizumab in subjects with unresectable solid tumors (KEYNOTE-603): an update. J. Immunother. Cancer https://doi.org/10.1136/itc-2020SITC2020.0798 (2020)

211. Burris, H. A. et al. A phase I multicenter study to assess the safety, tolerability, and immunogenicity of mRNA-4157 alone in patients with resected solid tumors and in combination with pembrolizumab in patients with unreshectable solid tumors. J. Clin. Oncol. 37, 2523 (2019)

212. Cafri, G. et al. mRNA vaccine-induced neoantigenspecific $T$ cell immunity in patients with gastrointestinal cancer. J. Clin. Invest. 130 5976-5988 (2020).

213. Reinhard, K. et al. An RNA vaccine drives expansion and efficacy of claudin-CAR-T cells against solid tumors. Science 367, 446-453 (2020).

214. Jimeno, A. et al. Abstract CT032: a phase 1/2, openlabel, multicenter, dose escalation and efficacy study of mRNA-2416, a lipid nanoparticle encapsulated mRNA encoding human $\mathrm{OX} 40 \mathrm{~L}$, for intratumoral injection alone or in combination with durvalumab for patients with advanced malignancies. Cancer Res. https://doi.org/10.1158/1538-7445.AM2020-CT032 (2020).

215. Bauer, T. et al. Abstract CT210: A Phase I, open-label, multicenter, dose escalation study of mRNA-2752, a lipid nanoparticle encapsulating mRNAs encoding human OX40L, IL-23, and IL-36\%, for intratumoral injection alone and in combination with immune checkpoint blockade. Cancer Res. https://doi.org/ 10.1158/1538-7445.AM2019-CT210 (2019).

216. Patel, M. R. et al. A phase I study of mRNA-2752, a lipid nanoparticle encapsulating mRNAs encoding human OX40L, IL-23, and IL-36y, for intratumoral (iTu) injection alone and in combination with durvalumab. J. Clin. Oncol. 38, 3092-3092 (2020).

217. Billingsley, M. M. et al. Ionizable lipid nanoparticlemediated mRNA delivery for human CAR T cell engineering. Nano Lett. 20, 1578-1589 (2020)

218. Kong, N. et al. Synthetic mRNA nanoparticle-mediated restoration of p53 tumor suppressor sensitizes p53 deficient cancers to mTOR inhibition. Sci. Transl Med. 11, eaaw1565 (2019)

219. Okumura, K. et al. Bax mRNA therapy using cationic liposomes for human malignant melanoma. J. Gene Med. 10, 910-917 (2008).

220. Islam, M. A. et al. Restoration of tumour-growth suppression in vivo via systemic nanoparticlemediated delivery of PTEN mRNA. Nat. Biomed. Eng. 2, 850-864 (2018)

221. An, D. et al. Systemic messenger RNA therapy as a treatment for methylmalonic acidemia. Cell Rep. 21 3548-3558 (2017).

222. Jiang, L. et al. Systemic messenger RNA as an etiological treatment for acute intermittent porphyria. Nat. Med. 24, 1899-1909 (2018).

223. Karadagi, A. et al. Systemic modified messenger RNA for replacement therapy in alpha 1-antitrypsin deficiency. Sci. Rep. 10, 7052 (2020).

224. Zhu, X. et al. Systemic mRNA therapy for the treatmen of Fabry disease: preclinical studies in wild-type mice, Fabry mouse model, and wild-type non-human primates. Am. J. Hum. Genet. 104, 625-637 (2019).

225. DeRosa, F. et al. Improved efficacy in a Fabry disease model using a systemic mRNA liver depot system as compared to enzyme replacement therapy. Mol. Ther. 27, 878-889 (2019).

226. Apgar, J. F. et al. Quantitative systems pharmacology model of hUGT 1 A 1-modRNA encoding for the UGT 1A1 enzyme to treat Crigler-Najjar syndrome type 1. CPT Pharmacomet. Syst. Pharmacol. 7, 404-412 (2018).

227. Connolly, B., Isaacs, C., Cheng, L., Asrani, K. H. \& Subramanian, R. R. SERPINA1 mRNA as a treatment for alpha-1 antitrypsin deficiency. J. Nucleic Acids 2018, 8247935 (2018)

228. An, D. et al. Long-term efficacy and safety of mRNA therapy in two murine models of methylmalonic acidemia. EBioMedicine 45, 519-528 (2019).

229. Truong, B. et al. Lipid nanoparticle-targeted mRNA therapy as a treatment for the inherited metabolic liver disorder arginase deficiency. Proc. Natl Acad. Sci. USA 116, 21150-21159 (2019).

230. Cao, J. et al. mRNA therapy improves metabolic and behavioral abnormalities in a murine model of citrin deficiency. Mol. Ther. 27, 1242-1251 (2019).

231. Roseman, D. S. et al. G6PC mRNA therapy positively regulates fasting blood glucose and decreases liver abnormalities in a mouse model of glycogen storage disease 1a. Mol. Ther. 26, 814-821 (2018).

232. Chen, C.-Y. et al. Treatment of hemophilia A using factor VIII messenger RNA lipid nanoparticles. Mol. Ther. Nucleic Acids 20, 534-544 (2020).

233. Ramaswamy, S. et al. Systemic delivery of factor IX messenger RNA for protein replacement therapy. Proc. Natl Acad. Sci. USA 114, E1941-E1950 (2017).

234. Liu-Chen, S., Connolly, B., Cheng, L., Subramanian, R. R. $\&$ Han, Z. mRNA treatment produces sustained expression of enzymatically active human ADAMTS13 in mice. Sci. Rep. 8, 7859 (2018)

235. Lescan, M. et al. De novo synthesis of elastin by exogenous delivery of synthetic modified mRNA into skin and elastin-deficient cells. Mol. Ther. Nucleic Acids 11, 475-484 (2018).

236. Blakney, A. K. et al. The skin you are in: designof-experiments optimization of lipid nanoparticle selfamplifying RNA formulations in human skin explants. ACS Nano 13, 5920-5930 (2019).

237. Miwa, T. Saito, H. \& Akita, H. Lipid nanoparticlesencapsulated brain-derived neurotrophic factor mRNA delivered through the round window niche in the cochleae of guinea pigs. Exp. Brain Res. 239 425-433 (2020)

238. Song, C.-Q. et al. Adenine base editing in an adult mouse model of tyrosinaemia. Nat. Biomed. Eng. 4 125-130 (2020)

239. Conway, A. et al. Non-viral delivery of zinc finger nuclease mRNA enables highly efficient in vivo genome editing of multiple therapeutic gene targets. Mol. Ther. 27, 866-877 (2019).

240. Finn, J. D. et al. A single administration of CRISPR/ Cas9 lipid nanoparticles achieves robust and persistent in vivo genome editing. Cell Rep. 22 2227-2235 (2018)

241. Magadum, A., Kaur, K. \& Zangi, L. mRNA-based protein replacement therapy for the heart. Mol. Ther. 27, 785-793 (2019)

242. Trepotec, Z., Lichtenegger, E., Plank, C., Aneja, M. K. \& Rudolph, C. Delivery of mRNA therapeutics for the treatment of hepatic diseases. Mol. Ther. 27, 794-802 (2019).

243. Sahu, I., Haque, A. A., Weidensee, B., Weinmann, P. \& Kormann, M. S. Recent developments in mRNA-based protein supplementation therapy to target lung diseases. Mol. Ther. 27, 803-823 (2019).

244. Thess, A. et al. Sequence-engineered mRNA without chemical nucleoside modifications enables an effective protein therapy in large animals. Mol. Ther. 23 1456-1464 (2015).

245. Wesselhoeft, R. A., Kowalski, P. S. \& Anderson, D. C. Engineering circular RNA for potent and stable translation in eukaryotic cells. Nat. Commun. 9, 2629 (2018).

246. Wesselhoeft, R. A. et al. RNA circularization diminishes immunogenicity and can extend translation duration in vivo. Mol. Cell 74, 508-520.e4 (2019).

247. McKinlay, C. J. et al. Charge-altering releasable transporters (CARTs) for the delivery and release of mRNA in living animals. Proc. Natl Acad. Sci. USA 114, E448-E456 (2017).

248. Chahal, J. S. et al. Dendrimer-RNA nanoparticles generate protective immunity against lethal Ebola, H1N1 influenza, and Toxoplasma gondii challenges with a single dose. Proc. Natl Acad. Sci. USA 113, E4133-E4142 (2016).

249. Yang, Z. et al. Large-scale generation of functional mRNA-encapsulating exosomes via cellular nanoporation. Nat. Biomed. Eng. 4, 69-83 (2020)

250. Fang, R. H., Kroll, A. V., Gao, W. \& Zhang, L. Cell membrane coating nanotechnology. Adv. Mater. 30 1706759 (2018).

251. Bangham, A., Standish, M. M. \& Watkins, J. C. Diffusion of univalent ions across the lamellae of swollen phospholipids. J. Mol. Biol. 13, 238-IN227 (1965).

252. Lockard, R. E. \& Lingrel, J. B. The synthesis of mouse hemoglobin chains in a rabbit reticulocyte cell-free system programmed with mouse reticulocyte 9S RNA Biochem. Biophys. Res. Commun. 37, 204-212 (1969).

253. Jirikowski, G. F., Sanna, P. P., Maciejewski-Lenoir, D. $\&$ Bloom, F. E. Reversal of diabetes insipidus in Brattleboro rats: intrahypothalamic injection of vasopressin mRNA. Science 255, 996-998 (1992).

\section{Acknowledgements}

This work was supported by the Maximizing Investigators' Research Award R35GM119679 from the National Institute of General Medical Sciences (to Y.D.) and grant EB000244 from the National Institute of Biomedical Imaging and Bioengineering (to R.L.). We acknowledge Y. Zhang for his help with chemical structures. All authors reviewed and edited the manuscript before submission.

\section{Author contributions}

X.H., T.Z, R.L. and Y.D. contributed to conceiving the structure, searching the literature and writing the Review. T.Z., R.L. and Y.D. reviewed and edited the manuscript.

\section{Competing interests}

Y.D is a scientific advisory board member of Oncorus, Inc. and serves as a consultant of Rubius Therapeutics. T.Z. is an employee of Moderna, Inc. R.L. is a founding scientific advisory board member of Alnylam and a founder and board member of Moderna, Inc. A list of entities with which R.L. is involved, compensated or uncompensated is provided in the supplementary information. X.H. declares no competing interests.

\section{Publisher's note}

Springer Nature remains neutral with regard to jurisdictional claims in published maps and institutional affiliations.

\section{Supplementary information}

The online version contains supplementary material available at https://doi.org/10.1038/s41578-021-00358-0.

\section{RELATED LINKS}

GMP standard tests:

drugsatfda_docs/nda/2018/210922Orig1s000ChemR.pdf mRNA-1893 interim phase I: https://investors.modernatx com/news-releases/news-release-details/modernamRNA-1944: https://investors.modernatx.com/node/7711/ pdf MRT5005: https://translatebio.gcs-web.com/news-releases/ results-phase-12-clinical-trial Storage of BNT162b2: https:// press-release/press-release-detail/ema-
storage-option-pfizer-biontech-vaccine

(c) Springer Nature Limited 2021, corrected publication 2021 